\title{
Multicriteria Decision Making Based on Archimedean Bonferroni Mean Operators of Hesitant Fermatean 2-Tuple Linguistic Terms
}

\author{
Huijuan Wang, Xin Wang $(\mathbb{D}$, and Lidong Wang $(\mathbb{D}$ \\ College of Science, Dalian Maritime University, Dalian 116026, China \\ Correspondence should be addressed to Xin Wang; xenawang@dlmu.edu.cn and Lidong Wang; ldwang@hotmail.com
}

Received 22 July 2019; Revised 30 October 2019; Accepted 15 November 2019; Published 20 December 2019

Academic Editor: Zhile Yang

Copyright (c) 2019 Huijuan Wang et al. This is an open access article distributed under the Creative Commons Attribution License, which permits unrestricted use, distribution, and reproduction in any medium, provided the original work is properly cited.

\begin{abstract}
The study is concerned with the representation and aggregation of complex uncertainty information. First, the concept of hesitant Fermatean 2-tuple linguistic sets (HF2TLSs) is introduced for characterizing an individual's imprecision preferences and assessing information by combining 2-tuple linguistic terms and Fermatean fuzzy sets. The advantage of hesitant Fermatean 2-tuple linguistic information is that it can handle higher levels of uncertainty and express the decision-makers' hesitancy. Second, we extend Bonferroni mean (BM) operators under the background of HF2TLSs for the sake of their application in information fusion and decision making. The Archimedean $t$-norm and $s$-norm- (ATS-) based hesitant Fermatean 2-tuple linguistic weighted Bonferroni mean (A-HF2TLWBM) operator and the ATS-based hesitant Fermatean 2-tuple linguistic weighted geometric Bonferroni mean (A-HF2TLWGBM) operator are developed by considering the interrelationship between any two variables. The main benefit of the proposed operators is that these operators deliver more complete and flexible results compared to existing methods. Moreover, some fundamental properties and special cases are examined by adjusting parameter values. Finally, an approach is designed as a support for handling decision making problems, and an example regarding investment selection is provided to demonstrate the practicality of the designed method with a detailed discussion of parameter influence and comparisons with the existing methods.
\end{abstract}

\section{Introduction}

Multicriteria decision making (MCDM) arises from decision theory, which can help us select the optimal solution from multiple possible alternatives and plays an indispensable role in various fields, such as the economy [1-3] and medicine [4]. MCDM is a complex process subject to external environmental influences and other subjective factors affecting decision-makers. In today's information-intensive conditions, imprecise information is increasing in volume and complexity, and some sound methods have been established. Linguistic variables, characterized by qualitative words or phrases, offer a natural and direct semantic description of the intuition and preferences of decision-makers [5]. For instance, when assessing teachers' levels, decision-makers tend to give their judgments in the form of linguistic terms, for example, "good," "fair," and "poor." Generally, state-of- the-art methods for representing linguistic variables are based on membership grades, symbolic computational methods, and combinations thereof.

Fuzzy set (FS) theory was introduced by Zadeh [6], in which linguistic terms are characterized in the manner of the membership degree expressed by a real number between $[0,1]$. To better reflect complex decision information in the form of linguistic terms, different types of fuzzy set-based methods have been developed. Orthopair fuzzy sets have been coined for describing imprecision information by introducing a pair of values $(\mu(z), v(z))$ in the unit interval, where $\mu(z)$ indicates the support degree (membership degree) and $\nu(z)$ records against degree (nonmembership degree) of $z$ belonging to a fuzzy concept. Compared with FS theory, the sum of $\mu(z)$ and $\nu(z)$ for element $z$ belonging to a fuzzy concept is no more than 1 in Atanassov's intuitionistic fuzzy sets (IFSs) [7]. In practice, a decision-maker 
may give his judgment information with $\mu(z)=0.7$ and $\nu(z)=0.6$, which is out of the application scope of IFS. Pythagorean fuzzy set (PFS), developed by Yager [8], is a new extension of IFS, in which the sum of squares of support degrees and against degrees is bounded by 1 . PFS is more versatile than IFS. Aiming to solve complex decision making problems, a series of extensions and generalizations of PFS have been explored [9-11]. With the increasing complexity involved in the decision making process, the description of abundant uncertainty still needs to be adequately addressed. If a decision-maker gives his preference using a pair of values with support degree 0.9 and against degree 0.5 , respectively, this is inconsistent with the definition of both IFS and PFS. Senapati and Yager [12, 13] coined the Fermatean fuzzy set (FFS) with its comparison measures. FFS can characterize more complex uncertain information by redefining the constraint condition $0 \leq\left(\mu_{F}(z)\right)^{3}+\left(\nu_{F}(z)\right)^{3} \leq 1$. In other words, IFS and PFS are two special forms of FFS, which means that the FFSs are able to handle higher levels of uncertainties.

Ordinal labels offer an easy and direct way of qualitative linguistic description for solving decision problems, and symbolic computational methods have been established on the basis of defining their operation laws. However, the original ordinal labels were imposed as the operational result, so information loss may be unavoidable during the operation process $[14,15]$. To address this problem, some efforts have been made by combining linguistic terms with real numbers (or membership degrees), which may also be better aligned with human decision making processes. Herrera and Martínez $[16,17]$ cultivated 2-tuple linguistic representation terms, whose element comprises a linguistic term and a real number. This approach possesses the characteristics of present fuzzy linguistic evaluation and also avoids the information loss problems of other fuzzy linguistic models. Subsequently, some decision making models have been developed based on 2-tuple linguistic models. Wang [18] built an appropriate approach with the aid of a 2tuple linguistic presentation model to assess the performance of a new product development project. Zhang et al. [19] established a consensus reaching approach under 2tuple linguistic judgment information and incomplete weight information.

In reality, because of the external factors of decision making situations and the internal cognitive preferences, some MCDM issues are very complicated and give rise to decision-makers' hesitancy. In order to depict and handle the decision-makers' hesitancy, Torra [20] coined the hesitant fuzzy set (HFS), taking multiple values in $[0,1]$ as the possible membership degrees. HFS is very effective in handling the human hesitation involved in the decisionmaking process. Recently, in the light of HFS, some extended models have been explored intensively. Zhou et al. [21] came up with the concept of the hesitant intuitionistic fuzzy set (HIFS) and proposed a decision making method by expressing complementary preference relationships for uncertain HIFS information. Zadeh [6] presented the thought of hesitant fuzzy linguistic term sets (HFLTSs). Rashid et al. [22] and Zhang et al. [23] introduced hesitant intuitionistic fuzzy linguistic term sets (HIFLTSs). Pythagorean 2-tuple linguistic sets studied by Wei et al. [24] are a combination of the PFSs and 2-tuple linguistic evaluation models for dealing with MCDM problems. Liu et al. [25] introduced the notion of Fermatean fuzzy linguistic term sets and developed the related TOPSIS method using different distance measures and aggregation operators. In order to handle higher levels of uncertainty and express decisionmakers' hesitancy, this study introduces hesitant Fermatean 2-tuple linguistic set (HF2TLS) by combining the FFS and 2tuple linguistic terms.

The fusion of uncertainty information can achieve some overall values of alternatives or group opinions in the decision making domain. To better reflect the preferences of decision-makers and the complexity of the decision making domain, a wide range of aggregation operators have been presented. The Bonferroni mean (BM) [26-28] operator is a mean-type aggregation approach, whose salient capability can depict the interrelationship of the input variables well. Some aggregation operators have been developed for highlighting specific roles or a relationship of aggregated variables within various MCDM situations. An in-depth study of Bonferroni mean operators was explored by Yager [29], and some generalized Bonferroni mean (GBM) operators were established to characterize the interrelationship among any three variables. Subsequently, Beliakov et al. [30] and Xia et al. [31-33] developed the dual GBM (DGBM) operators, which are a new generalization of the classical BM operator by considering the relatedness involved in all variables. These operators have been successfully extended to IFSs [32], PFSs [34], and HFSs [33]. In recent years, the Archimedean $t$-norm and $s$-norm (ATS) $[35,36]$ has been used as a generalization of most existing $t$-norms and $s$ norms, like algebraic and Einstein $t$-norm and $s$-norm [37], which play important roles in inducing multiple aggregation operators for fuzzy terms. Various applications indicate that ATS contributes to uncertain information fusion in complex decision making situations [38-40]. Lan et al. [38] employed the extended triangular co-norm to deal with linguistic information and proposed some aggregation operators. Xia et al. [39] introduced some new operations on IFSs based on ATS and presented some intuitionistic fuzzy aggregation operators. Tao et al. [40] proposed some new operational laws of 2-tuples based on ATS and gave some aggregation operators using the proposed rules.

Although Fermatean fuzzy linguistic terms provide a potential way to capture the cognitive uncertainty during making decisions, there exist some situations where humans may hesitate to express their preferences with a single variable. To better evaluate complex practical problems, it is necessary to combine hesitant fuzzy sets and Fermatean fuzzy linguistic terms to represent the subjective preferences with the higher levels of uncertainties. In this study, HF2TLSs are proposed, and their operational laws between HF2TLSs are developed. In order to allow HF2TLSs to handle higher levels of uncertainties and express the decision-makers' hesitancy, how to facilitate the aggregation of these HF2TLSs becomes a significant issue. We define two related aggregation operators of the ATS-based hesitant 
Fermatean 2-tuple linguistic weighted Bonferroni mean. These operators consider the interrelationship between any two variables. In addition, the established methods are two parameterized families of the operational laws and aggregation operators of HF2TLSs, respectively, which cover a wide range of particular forms that exhibit a more flexible way for adapting the specific needs of problem solving. To examine the practicality of the aggregation operators of HF2TLSs, we offer a procedure for solving ranking problems on the strength of the developed operators within hesitant Fermatean 2-tuple linguistic environments.

The structure of this study is as follows. Section 2 briefly reviewed some basic concepts including definition of FFSs, $\mathrm{BM}$ operator, and GBM operator. Section 3 introduces the definition of HF2TLSs. And some new operational rules and related theorems about HF2TLSs based on ATS are developed. Section 4 shows some aggregation operators and examines their properties and particular forms. In Section 5, we propose a ranking scheme under the hesitant Fermatean 2-tuple linguistic environment. In Section 6, an example with some detailed steps and comparisons is given to illustrate the practicality and flexibility of the aforementioned methods. The conclusion is presented in the final section.

\section{Preliminaries}

In the following, some fundamental definitions and functions are introduced.

Let $S f=\left\{s f_{0}, s f_{1}, s f_{2}, \ldots, s f_{g}\right\}$ be a collection of linguistic terms with odd cardinality, where $s f_{r}$ denotes a linguistic term with the order relationship $s f_{r}>s f_{t}$, if $r>t$, and the negation operator is neg $\left(s f_{r}\right)=s f_{g-r}[14]$. A 2-tuple linguistic method consists of $\left(s f_{r}, \alpha_{r}\right)$, where $s f_{r} \in S f$ and $\alpha_{r} \in[-0.5,0.5)$ denotes symbolic translation $[14,16,17]$.

Definition 1 (see [17]). Let $S f=\left\{s f_{r} \mid r=0,1, \ldots, g\right\}$ be a collection of linguistic terms, the function $\Delta$ is introduced to get $\beta \in[0,1]$ that is equivalent to a 2 -tuple linguistic term (2-TLT):

$$
\begin{aligned}
& \Delta:[0, g] \longrightarrow S f \times[-0.5,0.5), \\
& \Delta(\beta)=\left(s f_{r}, \alpha_{r}\right) \text { with } \begin{cases}s f_{r}, & r=\operatorname{round}(\beta), \\
\alpha_{r}=\beta-r, & \alpha_{r} \in[-0.5,0.5),\end{cases}
\end{aligned}
$$

where the output of the function "round" is a positive number that is close to $\beta . \Delta^{-1}$ is the inverse function of $\Delta$, and

$$
\begin{aligned}
& \Delta^{-1}: S f \times[-0.5,0.5) \longrightarrow[0, g] \\
& \Delta^{-1}:\left(s f_{r}, \alpha_{r}\right)=r+\alpha_{r}=\beta .
\end{aligned}
$$

From Definition 1, a linguistic term $s f_{r}$ has an equivalent element $\Delta\left(s f_{r}\right)=\left(s f_{r}, 0\right)$ in 2-tuple linguistic set by adding an element as symbolic translation.

Definition 2 (see [12]). A Fermatean fuzzy set $F$ in $Z$ is derived as

$$
F=\left\{\left\langle z, \mu_{F}(z), v_{F}(z)\right\rangle \mid z \in Z\right\}
$$

where $\mu_{F}(z): Z \longrightarrow[0,1]$ and $\nu_{F}(z): Z \longrightarrow[0,1]$ with the constraint condition

$$
0 \leq\left(\mu_{F}(z)\right)^{3}+\left(\nu_{F}(z)\right)^{3} \leq 1, \quad \text { for any } z \in Z,
$$

$\mu_{F}(z)$ and $\nu_{F}(z)$ denote the support (membership) degree and the against (nonmembership) degree of $z$ in the set $F$, respectively. And $z \in Z, \pi_{F}(z)=$ $\sqrt[3]{1-\left(\mu_{F}(z)\right)^{3}-\left(\nu_{F}(z)\right)^{3}}$ is identified as the indeterminacy degree of $z$ to $Z$. For convenience, Senapati and Yager called $\left\langle\mu_{F}(z), v_{F}(z)\right\rangle$ a Fermatean fuzzy number (FFN).

Definition 3 (see [33]). If $p, q>0$ and $\left\{u_{r} \mid r=1,2, \ldots, n\right\}$ is a set of nonnegative integers, the Bonferroni mean (BM) operator is given as follows:

$$
\operatorname{BM}^{p, q}\left(u_{1}, u_{2}, \ldots, u_{n}\right)=\left(\frac{1}{n(n-1)} \sum_{r, t=1, r \neq t}^{n} u_{r}^{p} u_{t}^{q}\right)^{1 /(p+q)} .
$$

Definition 4 (see [33]). If $p, q>0$ and $\left\{u_{r} \mid r=1,2, \ldots, n\right\}$ is a set of nonnegative integers, the geometric Bonferroni mean (GBM) operator is described as follows:

$$
\operatorname{GBM}^{p, q}\left(u_{1}, u_{2}, \ldots, u_{n}\right)=\frac{1}{p+q} \sum_{r, t=1, r \neq t}^{n}\left(\mathrm{pu}_{r}+\mathrm{qu}_{t}\right)^{1 / n(n-1)} \text {. }
$$

\section{Hesitant Fermatean Fuzzy 2-Tuple Linguistic Sets}

In order to handle higher levels of uncertainties and express the decision-makers' hesitancy, this section introduces hesitant Fermatean 2-tuple linguistic sets (HF2TLSs) on the basis of FFSs and 2-TLT to represent complex uncertainty information. We also deduce some operational laws of HF2TLSs information.

\subsection{Hesitant Fermatean Fuzzy 2-Tuple Linguistic Sets.}

Definition 5. A HF2TLS $\mathscr{F}$ on $Z$ is defined as follows:

$$
\mathscr{F}=\left\{\left\langle\left(s f_{\theta\left(\mathscr{F}_{r}\right)}, \alpha_{\mathscr{F}_{r}}\right), \mu_{\mathscr{F}_{r}}(z), v_{\mathscr{F}_{r}}(z)\right\rangle \mid\left(s f_{\theta\left(\mathscr{F}_{r}\right)}, \alpha_{\mathscr{F}_{r}}\right) \in S f\right\},
$$


where $s f_{\theta\left(\mathscr{F}_{r}\right)} \in S f, \alpha_{\mathscr{F}_{r}} \in[-0.5,0.5)$, and $\mu_{\mathscr{F}_{r}}(z), v_{\mathscr{F}_{r}}(z)$ are located in the unit interval $[0,1]$ with $0 \leq\left(\mu_{\mathscr{F}_{r}}\right.$ $(z))^{3}+\left(\nu_{\mathscr{F}_{r}}(z)\right)^{3} \leq 1 . \mu_{\mathscr{F}_{r}}(z)$ and $v_{\mathscr{F}_{r}}(z)$ denote the support degree and against degree of the element $\mathrm{z}$ belonging to $\left(s f_{\theta\left(\mathscr{F}_{r}\right)}, \alpha_{\mathscr{F}_{r}}\right)$, respectively. $1 \leq r \leq|\mathscr{F}|$ and $|\mathscr{F}|$ is the number of elements of $\mathscr{F}$. For convenience, $\left\langle\left(s f_{\theta\left(\mathscr{F}_{r}\right)}\right.\right.$, $\left.\left.\alpha_{\mathscr{F}_{r}}\right), \mu_{\mathscr{F}_{r}}(z), \nu_{\mathscr{F}_{r}}(z)\right\rangle$ is called a hesitant Fermatean 2-tuple linguistic number (HF2TLN).

Definition 6. Let $\mathscr{F}=\left\{\left\langle\left(s f_{\theta\left(\mathscr{F}_{r}\right.}, \alpha_{\mathscr{F}_{r}}\right), \mu_{\mathscr{F}_{r}}, v_{\mathscr{F}_{r}}\right\rangle\right\}, r=1,2$, ..., $|\mathscr{F}|$ be a HF2TLS; the score function of $\mathscr{F}$ is defined as follows:

$$
\operatorname{SC}(\mathscr{F})=\frac{\sum_{r=1}^{\mid \mathscr{F} 1}\left(\Delta^{-1}\left(s f_{\theta\left(\mathscr{F}_{r}\right)}, \alpha_{\mathscr{F}_{r}}\right) \times\left(\left(\mu_{\mathscr{F}_{r}}\right)^{3}-\left(\nu_{\mathscr{F}_{r}}\right)^{3}\right)\right)}{|\mathscr{F}|} .
$$

Definition 7. Let $\mathscr{F}=\left\{\left\langle\left(s f_{\theta\left(\mathscr{F}_{r}\right)}, \alpha_{\mathscr{F}_{r}}\right), \mu_{\mathscr{F}_{r}}, v_{\mathscr{F}_{r}}\right\rangle\right\}, r=1,2$, ..., $|\mathscr{F}|$ be a HF2TLS; the accuracy function of $\mathscr{F}$ is defined as follows:

$$
\operatorname{AC}(\mathscr{F})=\frac{\sum_{r=1}^{|\mathscr{F}|}\left(\Delta^{-1}\left(s f_{\theta\left(\mathscr{F}_{r}\right)}, \alpha_{\mathscr{F}_{r}}\right) \times\left(\left(\mu_{\mathscr{F}_{r}}\right)^{3}+\left(\nu_{\mathscr{F}_{r}}\right)^{3}\right)\right)}{|\mathscr{F}|} .
$$

Definition 8 . Let $\quad \mathscr{F}_{1}=\left\{\left\langle\left(s f_{\theta\left(\mathscr{F}_{r}\right)}, \alpha_{\mathscr{F}_{r}}\right), \mu_{\mathscr{F}_{r}}, \nu_{\mathscr{F}_{r}}\right\rangle\right\}, r=$ $1,2, \ldots,\left|\mathscr{F}_{1}\right|$ and $\mathscr{F}_{2}=\left\{\left\langle\left(s f_{\theta\left(\mathscr{F}_{t}\right)}, \alpha_{\mathscr{F}_{t}}\right), \mu_{\mathscr{F}_{t}}, v_{\mathscr{F}_{t}}\right\rangle\right\}, t=1,2$, $\ldots,\left|\mathscr{F}_{2}\right|$ be two HF2TLSs; then, the order relationships between $\mathscr{F}_{1}$ and $\mathscr{F}_{2}$ are described as follows:

(1) If $\operatorname{SC}\left(\mathscr{F}_{1}\right)<\operatorname{SC}\left(\mathscr{F}_{2}\right)$, then $\mathscr{F}_{1}<\mathscr{F}_{2}$

(2) If $\operatorname{SC}\left(\mathscr{F}_{1}\right)>\operatorname{SC}\left(\mathscr{F}_{2}\right)$, then $\mathscr{F}_{1}>\mathscr{F}_{2}$

(3) When $\operatorname{SC}\left(\mathscr{F}_{1}\right)=\operatorname{SC}\left(\mathscr{F}_{2}\right)$,

(a) $\mathrm{AC}\left(\mathscr{F}_{1}\right)=\mathrm{AC}\left(\mathscr{F}_{2}\right)$, and $\mathscr{F}_{1} \sim \mathscr{F}_{2}$

(b) $\mathrm{AC}\left(\mathscr{F}_{1}\right)<\mathrm{AC}\left(\mathscr{F}_{2}\right)$, and $\mathscr{F}_{1}<\mathscr{F}_{2}$

(c) $\mathrm{AC}\left(\mathscr{F}_{1}\right)>\mathrm{AC}\left(\mathscr{F}_{2}\right)$, and $\mathscr{F}_{1}>\mathscr{F}_{2}$

3.2. New Operational Laws for HF2TLSs. The $t$-norm and $s$ norm are two kinds of binary operations used in probabilistic metrics and fuzzy logic, which generalizes intersection operation and conjunction operation. An Archimedean $t$-norm is defined as $T(c, d)=\zeta^{-1}(\zeta(c)+\zeta(d))$, where $\zeta$ is a strictly continuous decreasing function $\zeta:[0,1] \longrightarrow[0, \infty]$ such that $\zeta(1)=0$. Its dual function $s$-norm can be externalized as $S(c, d)=\chi^{-1}(\chi(c)+\chi(d))$ with $\chi(c)=\zeta(1-c)$. By using a different mapping $T:[0,1] \times[0,1] \longrightarrow[0,1]$, multiple different operators can be derived for handling fuzzy term aggregation problems. ATS was incorporated into 2-tuple linguistic environments by Tao et al. [40], and some ATSbased operational laws for intuitionistic fuzzy numbers were developed by Xia et al. [31-33]. Enlightened by these works, we deduce the following operational laws of HF2TLSs information.

Definition 9. Let $\mathscr{F}_{1}=\left\{\left\langle\left(s f_{\theta\left(\mathscr{F}_{r}\right)}, \alpha_{\mathscr{F}_{r}}\right), \mu_{\mathscr{F}_{r}}, v_{\mathscr{F}_{r}}\right\rangle\right\}, r=1,2$, $\ldots,\left|\mathscr{F}_{1}\right| \quad$ and $\quad \mathscr{F}_{2}=\left\{\left\langle\left(s f_{\theta\left(\mathscr{F}_{t}\right.}, \alpha_{\mathscr{F}_{t}}\right), \mu_{\mathscr{F}_{t}}, \nu_{\mathscr{F}_{t}}\right\rangle\right\}, t=1,2$, $\ldots,\left|\mathscr{F}_{2}\right|$ be any two HF2TLSs; then, some operational laws are defined as follows $\left(\lambda>0\right.$ and $\left.\Delta_{\mathscr{F}_{r}}^{-1}=\Delta^{-1}\left(s f_{\theta\left(\mathscr{F}_{r}\right)}, \alpha_{\mathscr{F}_{r}}\right)\right)$ :

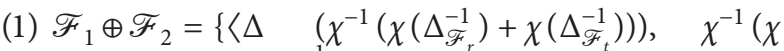
$\left.\left.\left(\mu_{\mathscr{F}_{r}}\right)+\chi\left(\mu_{\mathscr{F}_{t}}\right)\right), \zeta^{-1}\left(\zeta\left(\nu_{\mathscr{F}_{r}}\right)+\zeta\left(\nu_{\mathscr{F}_{t}}\right)\right)\right\rangle\left.\right|^{t}\left\langle\left(s f_{\theta\left(\mathscr{F}_{r}\right)}\right.\right.$, $\left.\left.\alpha_{\mathscr{F}_{r}}\right), \mu_{\mathscr{F}_{r}}, v_{\mathscr{F}_{r}}\right\rangle \in \mathscr{F}_{1},\left\langle\left(s f_{\theta\left(\mathscr{F}_{t}\right)}^{r}, \alpha_{\mathscr{F}_{t}}\right), \mu_{\mathscr{F}_{t}}, v_{\mathscr{F}_{t}}\right\rangle \in$ $\left.\mathscr{F}_{2}\right\}$

(2) $\mathscr{F}_{1} \otimes \mathscr{F}_{2}=\left\{\left\langle\Delta\left(\zeta^{-1}\left(\zeta\left(\triangle_{\mathscr{F}_{r}}^{-1}\right)+\zeta\left(\triangle_{\mathscr{F}_{t}}^{-1}\right)\right)\right), \zeta^{-1}(\zeta\right.\right.$ $\left(\mu_{\mathscr{F}}\right)$ $\left.\left.+\zeta\left(\mu_{\mathscr{F}_{t}}\right)\right), \chi^{-1}\left(\chi\left(\nu_{\mathscr{F}_{r}}\right)+\chi\left(\nu_{\mathscr{F}_{t}}\right)\right)\right\rangle \mid\left\langle\left(s f_{\theta\left(\mathscr{F}_{r}\right)}, \alpha_{\mathscr{F}_{r}}\right)\right.$, $\left.\left.\mu_{\mathscr{F}_{r}}, \nu_{\mathscr{F}_{r}}\right\rangle \in \mathscr{F}_{1},\left\langle\left(s f_{\theta}\left(\mathscr{F}_{t}\right), \alpha_{\mathscr{F}_{t}}\right), \mu_{\mathscr{F}_{t}}, \nu_{\mathscr{F}_{t}}\right\rangle \in \mathscr{F}_{2}\right\}$

(3) $\lambda \mathscr{F}_{1}=\left\{\left\langle\Delta\left(\chi^{-1}\left(\lambda \chi\left(\Delta_{\mathscr{F}_{r}}^{-1}\right)\right)\right), \chi^{-1}\left(\lambda \chi\left(\mu_{\mathscr{F}_{r}}\right)\right), \zeta^{-1}\right.\right.$ $\left.\left.\left(\lambda \zeta\left(\nu_{\mathscr{F}_{r}}\right)\right)\right\rangle \mid\left\langle\left(s f_{\theta\left(\mathscr{F}_{r}\right)}, \alpha_{\mathscr{F}_{r}}\right), \mu_{\mathscr{F}_{r}}, \nu_{\mathscr{F}_{r}}\right\rangle \in \mathscr{F}_{1}\right\}$

(4) $\mathscr{F}_{1}^{\lambda}=\left\{\left\langle\Delta\left(\zeta^{-1}\left(\lambda \zeta\left(\triangle_{\mathscr{F}_{r}}^{-1}\right)\right)\right), \zeta^{-1}\left(\lambda \zeta\left(\mu_{\mathscr{F}_{r}}\right)\right), \chi^{-1}(\lambda \chi\right.\right.$ $\left.\left.\left.\left(\nu_{\mathscr{F}_{r}}\right)\right)\right\rangle \mid\left\langle\left(s f_{\theta\left(\mathscr{F}_{r}\right)}, \alpha_{\mathscr{F}_{r}}\right), \mu_{\mathscr{F}_{r}}, \nu_{\mathscr{F}_{r}}\right\rangle \in \mathscr{F}_{1}\right\}$

In the sequel, some special operational laws are obtained by taking different forms of $\zeta(c)$ and their dual function $\chi$ $(c)=\zeta(1-c) . \quad$ Meanwhile, $\quad\left\langle\left(s f_{\theta\left(\mathscr{F}_{r}\right)}, \alpha_{\mathscr{F}_{r}}\right), \mu_{\mathscr{F}_{r}}, \nu_{\mathscr{F}_{r}}\right\rangle \epsilon$ $\mathscr{F}_{1}$ and $\left\langle\left(s f_{\theta\left(\mathscr{F}_{t}\right)}, \alpha_{\mathscr{F}_{t}}\right), \mu_{\mathscr{F}_{t}}, \nu_{\mathscr{F}_{t}}\right\rangle \in \mathscr{F}_{2}$ are simplified, so it is omitted during the operation process of HF2TLSs.

Remark 1. Let $\zeta(c)=-\log c$ and $\chi(c)=-\log (1-c)$; then, the operational laws defined by Definition 9 can be read as the following equations:
(1) $\mathscr{F}_{1} \oplus \mathscr{F}_{2}=\left\{\left\langle\Delta\left(\Delta_{\mathscr{F}_{r}}^{-1}+\Delta_{\mathscr{F}_{t}}^{-1}-\Delta_{\mathscr{F}_{r}}^{-1} \cdot \Delta_{\mathscr{F}_{t}}^{-1}\right)\right.\right.$, $\left.\left.\mu_{\mathscr{F}_{r}}+\mu_{\mathscr{F}_{t}}-\mu_{\mathscr{F}_{r}} \mu_{\mathscr{F}_{t}}, \nu_{\mathscr{F}_{r}} \nu_{\mathscr{F}_{t}}\right\rangle\right\}$
(2) $\mathscr{F}_{1} \otimes \mathscr{F}_{2}=\left\{\left\langle\Delta\left(\Delta_{\mathscr{F}_{r}}^{-1} \cdot \Delta_{\mathscr{F}_{t}}^{-1}\right), \mu_{\mathscr{F}_{r}} \mu_{\mathscr{F}_{t}}, \nu_{\mathscr{F}_{r}}+\nu_{\mathscr{F}_{t}}-\right.\right.$ $\left.\left.\nu_{\mathscr{F}_{r}} \nu_{\mathscr{F}_{t}}\right\rangle\right\}$
(3) $\lambda \mathscr{F}_{1}=\left\{\left\langle\Delta\left(1-\left(1-\Delta_{\mathscr{F}_{r}}^{-1}\right)^{\lambda}\right), 1-\left(1-\mu_{\mathscr{F}_{r}}\right)^{\lambda}, \nu_{\mathscr{F}_{r}}^{\lambda}\right\rangle\right\}$
(4) $\mathscr{F}_{1}^{\lambda}=\left\{\left\langle\Delta\left(\left(\Delta_{\mathscr{F}_{r}}^{-1}\right)^{\lambda}\right), \mu_{\mathscr{F}_{r}}^{\lambda}, 1-\left(1-v_{\mathscr{F}_{r}}\right)^{\lambda}\right\rangle\right\}$

Remark 2. If $\zeta(c)=\log ((2-c) / c)$ and $\chi(c)=\log ((2-$ $(1-c)) /(1-c))$, then the operational laws via the Einstein $t$ norm and $s$-norm can be obtained: 


$$
\begin{aligned}
& \mathscr{F}_{1} \oplus \mathscr{F}_{2}=\left\{\left\langle\Delta\left(\frac{\Delta_{\mathscr{F}_{r}}^{-1}+\Delta_{\mathscr{F}_{t}}^{-1}}{1+\Delta_{\mathscr{F}_{r}}^{-1} \cdot \Delta_{\mathscr{F}_{t}}^{-1}}\right), \frac{\mu_{\mathscr{F}_{r}}+\mu_{\mathscr{F}_{t}}}{1+\mu_{\mathscr{F}_{r}} \mu_{\mathscr{F}_{t}}}, \frac{v_{\mathscr{F}_{r}} \nu_{\mathscr{F}_{t}}}{1+\left(1-v_{\mathscr{F}_{r}}\right)\left(1-v_{\mathscr{F}_{t}}\right)}\right\rangle\right\} \\
& \mathscr{F}_{1} \otimes \mathscr{F}_{2}=\left\{\left\langle\Delta\left(\frac{\Delta_{\mathscr{F}_{r}}^{-1} \cdot \Delta_{\mathscr{F}_{t}}^{-1}}{1-\left(1-\Delta_{\mathscr{F}_{r}}^{-1}\right)\left(1-\Delta_{\mathscr{F}_{t}}^{-1}\right)}\right), \frac{\mu_{\mathscr{F}_{r}} \mu_{\mathscr{F}_{t}}}{1-\left(1-\mu_{\mathscr{F}_{r}}\right)\left(1-\mu_{\mathscr{F}_{t}}\right)}, \frac{v_{\mathscr{F}_{r}}+\nu_{\mathscr{F}_{t}}}{1+v_{\mathscr{F}_{r}} \nu_{\mathscr{F}_{t}}}\right\rangle\right\} ; \\
& \lambda \mathscr{F}_{1}=\left\{\left\langle\Delta\left(\frac{\left(1+\Delta_{\mathscr{F}_{r}}^{-1}\right)^{\lambda}-\left(1-\Delta_{\mathscr{F}_{r}}^{-1}\right)^{\lambda}}{\left(1+\Delta_{\mathscr{F}_{r}}^{-1}\right)^{\lambda}+\left(1-\Delta_{\mathscr{F}_{r}}^{-1}\right)^{\lambda}}\right), \frac{\left(1+\mu_{\mathscr{F}_{r}}\right)^{\lambda}-\left(1-\mu_{\mathscr{F}_{r}}\right)^{\lambda}}{\left(1+\mu_{\mathscr{F}_{r}}\right)^{\lambda}+\left(1-\mu_{\mathscr{F}_{r}}\right)^{\lambda}} \frac{2 \nu_{\mathscr{F}_{r}}^{\lambda}}{\left(2-\nu_{\mathscr{F}_{r}}\right)^{\lambda}+\nu_{\mathscr{F}_{r}}^{\lambda}}\right\rangle\right\} ; \\
& \mathscr{F}_{1}^{\lambda}=\left\{\left\langle\Delta\left(\frac{2\left(\Delta_{\mathscr{F}_{r}}^{-1}\right)^{\lambda}}{\left(2-\Delta_{\mathscr{F}_{r}}^{-1}\right)^{\lambda}+\left(\Delta_{\mathscr{F}_{r}}^{-1}\right)^{\lambda}}\right), \frac{2 \mu_{\mathscr{F}_{r}}^{\lambda}}{\left(2-\mu_{\mathscr{F}_{r}}\right)^{\lambda}+\mu_{\mathscr{F}_{r}}^{\lambda}}, \frac{\left(1+\nu_{\mathscr{F}_{r}}\right)^{\lambda}-\left(1-\nu_{\mathscr{F}_{r}}\right)^{\lambda}}{\left(1+\nu_{\mathscr{F}_{r}}\right)^{\lambda}+\left(1-\nu_{\mathscr{F}_{r}}\right)^{\lambda}}\right\rangle\right\} .
\end{aligned}
$$

Remark 3. If $\zeta(c)=\log ((\varepsilon+((1-\varepsilon) c)) / c), \chi(c)=\log ((1-$ $((1-\varepsilon) c)) /(1-c))$, and $\varepsilon>0$, then the operational laws via the Hamacher $t$-norm and $s$-norm can be obtained:

$$
\begin{aligned}
& \mathscr{F}_{1} \oplus \mathscr{F}_{2}=\left\{\left\langle\Delta\left(\frac{\Delta_{\mathscr{F}_{r}}^{-1}+\Delta_{\mathscr{F}_{t}}^{-1}-(1-\varepsilon) \cdot \Delta_{\mathscr{F}_{r}}^{-1} \cdot \Delta_{\mathscr{F}_{t}}^{-1}}{1-(1-\varepsilon) \cdot \Delta_{\mathscr{F}_{r}}^{-1} \cdot \Delta_{\mathscr{F}_{t}}^{-1}}\right), \frac{\mu_{\mathscr{F}_{r}}+\mu_{\mathscr{F}_{t}}-(1-\varepsilon) \mu_{\mathscr{F}_{r}} \mu_{\mathscr{F}_{t}}}{1-(1-\varepsilon) \mu_{\mathscr{F}_{r}} \mu_{\mathscr{F}_{t}}}, \frac{v_{\mathscr{F}_{r}} \nu_{\mathscr{F}_{t}}}{\varepsilon+(1-\varepsilon)\left(\nu_{\mathscr{F}_{r}}+\nu_{\mathscr{F}_{t}}-\nu_{\mathscr{F}_{r}} \nu_{\mathscr{F}_{t}}\right)}\right\rangle\right\} \\
& \mathscr{F}_{1} \otimes \mathscr{F}_{2}=\left\{\left\langle\Delta\left(\frac{\Delta_{\mathscr{F}_{r}}^{-1} \cdot \Delta_{\mathscr{F}_{t}}^{-1}}{\varepsilon+(1-\varepsilon)\left(\Delta_{\mathscr{F}_{r}}^{-1}+\Delta_{\mathscr{F}_{t}}^{-1}-\Delta_{\mathscr{F}_{r}}^{-1} \cdot \Delta_{\mathscr{F}_{t}}^{-1}\right)}\right), \frac{\mu_{\mathscr{F}_{r}} \mu_{\mathscr{F}_{t}}}{\varepsilon+(1-\varepsilon)\left(\mu_{\mathscr{F}_{r}}+\mu_{\mathscr{F}_{t}}-\mu_{\mathscr{F}_{r}} \mu_{\mathscr{F}_{t}}\right)}, \frac{\nu_{\mathscr{F}_{r}}+\nu_{\mathscr{F}_{t}}-(1-\varepsilon) \nu_{\mathscr{F}_{r}} \nu_{\mathscr{F}_{t}}}{1-(1-\varepsilon) \nu_{\mathscr{F}_{r}} \nu_{\mathscr{F}_{t}}}\right\rangle\right\} ;
\end{aligned}
$$

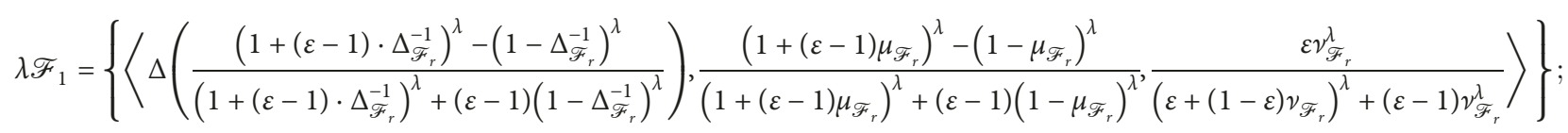

$$
\begin{aligned}
& \mathscr{F}_{1}^{\lambda}=\left\{\left\langle\Delta\left(\frac{\varepsilon\left(\Delta_{\mathscr{F}_{r}}^{-1}\right)^{\lambda}}{\left(\varepsilon+(1-\varepsilon) \cdot \Delta_{\mathscr{F}_{r}}^{-1}\right)^{\lambda}+(\varepsilon-1)\left(\Delta_{\mathscr{F}_{r}}^{-1}\right)^{\lambda}}\right), \frac{\varepsilon \mu_{\mathscr{F}_{r}}^{\lambda}}{\left(\varepsilon+(1-\varepsilon) \mu_{\mathscr{F}_{r}}\right)^{\lambda}+(\varepsilon-1) \mu_{\mathscr{F}_{r}}^{\lambda}}, \frac{\left(1+(\varepsilon-1) \nu_{\mathscr{F}_{r}}\right)^{\lambda}-\left(1-v_{\mathscr{F}_{r}}\right)^{\lambda}}{\left(1+(\varepsilon-1) \nu_{\mathscr{F}_{r}}\right)^{\lambda}+(\varepsilon-1)\left(1-\nu_{\mathscr{F}_{r}}\right)^{\lambda}}\right\rangle\right\} .
\end{aligned}
$$

Remark 4. Let $\zeta(c)=\log \left((\varepsilon-1) /\left(\varepsilon^{c}-1\right)\right), \chi(c)=\log ((\varepsilon-$ $\left.1) /\left(\varepsilon^{1-c}-1\right)\right)$, and $\varepsilon>1$, then the operational laws via the Frank $t$-norm and $s$-norm can be obtained:

$$
\begin{aligned}
& \mathscr{F}_{1} \oplus \mathscr{F}_{2}=\left\{\left\langle\Delta\left(\log _{\varepsilon}\left(\frac{\varepsilon(\varepsilon-1)}{\left(\varepsilon^{\Delta_{\mathscr{F}_{r}}^{-1}-1}-1\right)\left(\varepsilon^{\Delta_{\mathscr{F}_{t}}^{-1}-1}-1\right)+(\varepsilon-1)}\right)\right), \log _{\varepsilon}\left(\frac{\varepsilon(\varepsilon-1)}{\left(\varepsilon^{\mu_{\mathscr{F}_{r}-1}}-1\right)\left(\varepsilon^{\mu_{\mathscr{F}_{t}-1}}-1\right)+(\varepsilon-1)}\right), \log _{\varepsilon}\left(1+\frac{\left(\varepsilon^{\nu_{\mathscr{F}_{r}}}-1\right)\left(\varepsilon^{\nu_{\mathscr{F}_{t}}}-1\right)}{(\varepsilon-1)}\right)\right\rangle\right\} \\
& \mathscr{F}_{1} \otimes \mathscr{F}_{2}=\left\{\left\langle\Delta\left(\log _{\varepsilon}\left(1+\frac{\left(\varepsilon^{\Delta \Delta_{\mathscr{F}_{r}}^{-1}}-1\right)\left(\varepsilon^{\Delta_{\mathscr{F}_{t}}^{-1}}-1\right)}{(\varepsilon-1)}\right)\right), \log _{\varepsilon}\left(1+\frac{\left(\varepsilon^{\mu_{\mathscr{F}_{r}}}-1\right)\left(\varepsilon^{\mu_{\mathscr{F}_{t}}}-1\right)}{(\varepsilon-1)}\right), \log _{\varepsilon}\left(\frac{\varepsilon(\varepsilon-1)}{\left(\varepsilon^{\nu_{\mathscr{F}_{r}-1}}-1\right)\left(\varepsilon^{\nu_{\mathscr{F}_{t}-1}}-1\right)+(\varepsilon-1)}\right)\right\rangle\right\} \\
& \lambda \mathscr{F}_{1}=\left\{\left\langle\Delta\left(1-\log _{\varepsilon}\left(1+\frac{\left(\varepsilon^{1-\Delta_{\mathscr{F}_{r}}^{-1}}-1\right)^{\lambda}}{(\varepsilon-1)^{\lambda-1}}\right)\right), 1-\log _{\varepsilon}\left(1+\frac{\left(\varepsilon^{1-\mu_{\mathscr{F}_{r}}}-1\right)^{\lambda}}{(\varepsilon-1)^{\lambda-1}}\right), \log _{\varepsilon}\left(1+\frac{\left(\varepsilon^{\nu_{\mathscr{F}_{r}}}-1\right)^{\lambda}}{(\varepsilon-1)^{\lambda-1}}\right)\right\rangle\right\} ; \\
& \mathscr{F}_{1}^{\lambda}=\left\{\left\langle\Delta\left(\log _{\varepsilon}\left(1+\frac{\left(\varepsilon^{\Delta_{\mathscr{F}_{r}}^{-1}}-1\right)^{\lambda}}{(\varepsilon-1)^{\lambda-1}}\right)\right), \log _{\varepsilon}\left(1+\frac{\left(\varepsilon^{\mu_{\mathscr{F}_{r}}}-1\right)^{\lambda}}{(\varepsilon-1)^{\lambda-1}}\right), 1-\log _{\varepsilon}\left(1+\frac{\left(\varepsilon^{1-v_{\mathscr{F}_{r}}}-1\right)^{\lambda}}{(\varepsilon-1)^{\lambda-1}}\right)\right\rangle\right\} .
\end{aligned}
$$


Theorem 1. Let $\mathscr{F}_{1}$ and $\mathscr{F}_{2}$ are two HF2TLSs, then the following equations hold $\left(\lambda, \lambda_{1}, \lambda_{2}>0\right)$.

(1) $\mathscr{F}_{1} \oplus \mathscr{F}_{2}=\mathscr{F}_{2} \oplus \mathscr{F}_{1}$

(2) $\mathscr{F}_{1} \otimes \mathscr{F}_{2}=\mathscr{F}_{2} \otimes \mathscr{F}_{1}$

(3) $\lambda\left(\mathscr{F}_{1} \oplus \mathscr{F}_{2}\right)=\lambda \mathscr{F}_{1} \oplus \lambda \mathscr{F}_{2}$
(4) $\left(\mathscr{F}_{1} \otimes \mathscr{F}_{2}\right)^{\lambda}=\mathscr{F}_{1}^{\lambda} \otimes \mathscr{F}_{2}^{\lambda}$

(5) $\left(\lambda_{1}+\lambda_{2}\right) \mathscr{F}_{1}=\lambda_{1} \mathscr{F}_{1} \oplus \lambda_{2} \mathscr{F}_{1}$

(6) $\mathscr{F}_{1}^{\lambda_{1}+\lambda_{2}}=\mathscr{F}_{1}^{\lambda_{1}} \otimes \mathscr{F}_{1}^{\lambda_{2}}$

Proof

$$
\begin{aligned}
\mathscr{F}_{1} \oplus \mathscr{F}_{2} & =\left\{\left\langle\Delta\left(\chi^{-1}\left(\chi\left(\Delta_{\mathscr{F}_{r}}^{-1}\right)+\chi\left(\Delta_{\mathscr{F}_{t}}^{-1}\right)\right)\right), \chi^{-1}\left(\chi\left(\mu_{\mathscr{F}_{r}}\right)+\chi\left(\mu_{\mathscr{F}_{t}}\right)\right), \zeta^{-1}\left(\zeta\left(\nu_{\mathscr{F}_{r}}\right)+\zeta\left(\nu_{\mathscr{F}_{t}}\right)\right)\right\rangle\right\} \\
& =\left\{\left\langle\Delta\left(\chi^{-1}\left(\chi\left(\Delta_{\mathscr{F}_{t}}^{-1}\right)+\chi\left(\Delta_{\mathscr{F}_{r}}^{-1}\right)\right)\right), \chi^{-1}\left(\chi\left(\mu_{\mathscr{F}_{t}}\right)+\chi\left(\mu_{\mathscr{F}_{r}}\right)\right), \zeta^{-1}\left(\zeta\left(\nu_{\mathscr{F}_{t}}\right)+\zeta\left(\nu_{\mathscr{F}_{r}}\right)\right)\right\rangle\right\} \\
& =\mathscr{F}_{2} \oplus \mathscr{F}_{1} \cdot \\
\mathscr{F}_{1} \otimes \mathscr{F}_{2} & =\left\{\left\langle\Delta\left(\zeta^{-1}\left(\zeta\left(\Delta_{\mathscr{F}_{r}}^{-1}\right)+\zeta\left(\Delta_{\mathscr{F}_{t}}^{-1}\right)\right)\right), \zeta^{-1}\left(\zeta\left(\mu_{\mathscr{F}_{r}}\right)+\zeta\left(\mu_{\mathscr{F}_{t}}\right)\right), \chi^{-1}\left(\chi\left(\nu_{\mathscr{F}_{r}}\right)+\chi\left(\nu_{\mathscr{F}_{t}}\right)\right)\right\rangle\right\} \\
& =\left\{\left\langle\Delta\left(\zeta^{-1}\left(\zeta\left(\Delta_{\mathscr{F}_{t}}^{-1}\right)+\zeta\left(\Delta_{\mathscr{F}_{r}}^{-1}\right)\right)\right), \zeta^{-1}\left(\zeta\left(\mu_{\mathscr{F}_{t}}\right)+\zeta\left(\mu_{\mathscr{F}_{r}}\right)\right), \chi^{-1}\left(\chi\left(\nu_{\mathscr{F}_{t}}\right)+\chi\left(\nu_{\mathscr{F}_{r}}\right)\right)\right\rangle\right\} \\
& =\mathscr{F}_{2} \otimes \mathscr{F}_{1} . \\
\lambda\left(\mathscr{F}_{1} \oplus \mathscr{F}_{2}\right)= & \left\{\left\langle\Delta\left(\chi^{-1}\left(\lambda \chi\left(\chi^{-1}\left[\chi\left(\Delta_{\mathscr{F}_{r}}^{-1}\right)+\chi\left(\Delta_{\mathscr{F}_{t}}^{-1}\right)\right]\right)\right)\right), \chi^{-1}\left(\lambda \chi\left(\chi^{-1}\left[\chi\left(\mu_{\mathscr{F}_{r}}\right)+\chi\left(\mu_{\mathscr{F}_{t}}\right)\right]\right)\right), \zeta^{-1}\left(\lambda \zeta\left(\zeta^{-1}\left[\zeta\left(\nu_{\mathscr{F}_{r}}\right)+\zeta\left(\nu_{\mathscr{F}_{t}}\right)\right]\right)\right)\right\rangle\right\} \\
& =\left\{\left\langle\Delta\left(\chi^{-1}\left(\lambda \chi\left(\Delta_{\mathscr{F}_{r}}^{-1}\right)+\lambda \chi\left(\Delta_{\mathscr{F}_{t}}^{-1}\right)\right)\right), \chi^{-1}\left(\lambda \chi\left(\mu_{\mathscr{F}_{r}}\right)+\lambda \chi\left(\mu_{\mathscr{F}_{t}}\right)\right), \zeta^{-1}\left(\lambda \zeta\left(\nu_{\mathscr{F}_{r}}\right)+\lambda \zeta\left(\nu_{\mathscr{F}_{t}}\right)\right)\right\rangle\right\} \\
\lambda \mathscr{F}_{1} \oplus \lambda \mathscr{F}_{2}= & \left\{\left\langle\Delta\left(\chi^{-1}\left(\chi\left(\chi^{-1}\left[\lambda \chi\left(\Delta_{\mathscr{F}_{r}}^{-1}\right)+\lambda \chi\left(\Delta_{\mathscr{F}_{t}}\right)\right]\right)\right)\right), \chi^{-1}\left(\chi\left(\chi^{-1}\left[\lambda \chi\left(\mu_{\mathscr{F}_{r}}\right)+\lambda \chi\left(\mu_{\mathscr{F}_{t}}\right)\right]\right)\right), \zeta^{-1}\left(\zeta\left(\zeta^{-1}\left[\lambda \zeta\left(\nu_{\mathscr{F}_{r}}\right)+\lambda \zeta\left(\nu_{\mathscr{F}_{t}}\right)\right]\right)\right)\right\rangle\right\} \\
& =\left\{\left\langle\Delta\left(\chi^{-1}\left(\lambda \chi\left(\Delta_{\mathscr{F}_{r}}^{-1}\right)+\lambda \chi\left(\Delta_{\mathscr{F}_{t}}^{-1}\right)\right)\right), \chi^{-1}\left(\lambda \chi\left(\mu_{\mathscr{F}_{r}}\right)+\lambda \chi\left(\mu_{\mathscr{F}_{t}}\right)\right), \zeta^{-1}\left(\lambda \zeta\left(\nu_{\mathscr{F}_{r}}\right)+\lambda \zeta\left(\nu_{\mathscr{F}_{t}}\right)\right)\right\rangle\right\} .
\end{aligned}
$$

$\operatorname{So} \lambda\left(\mathscr{F}_{1} \oplus \mathscr{F}_{2}\right)=\lambda \mathscr{F}_{1} \oplus \lambda \mathscr{F}_{2}$ holds.

$$
\begin{aligned}
\left(\mathscr{F}_{1} \otimes \mathscr{F}_{2}\right)^{\lambda}= & \left\{\left\langle\Delta\left(\zeta^{-1}\left(\lambda \zeta\left(\zeta^{-1}\left(\zeta\left(\Delta_{\mathscr{F}_{r}}^{-1}\right)+\zeta\left(\Delta_{\mathscr{F}_{t}}^{-1}\right)\right)\right)\right)\right), \zeta^{-1}\left(\lambda \zeta\left(\zeta^{-1}\left(\zeta\left(\mu_{\mathscr{F}_{r}}\right)+\zeta\left(\mu_{\mathscr{F}_{t}}\right)\right)\right)\right), \chi^{-1}\left(\lambda \chi\left(\chi^{-1}\left(\chi\left(\nu_{\mathscr{F}_{r}}\right)+\chi\left(v_{\mathscr{F}_{t}}\right)\right)\right)\right)\right\rangle\right\} \\
= & \left\{\left\langle\Delta\left(\zeta^{-1}\left(\lambda \zeta\left(\Delta_{\mathscr{F}_{r}}^{-1}\right)+\lambda \zeta\left(\Delta_{\mathscr{F}_{t}}^{-1}\right)\right)\right), \zeta^{-1}\left(\lambda \zeta\left(\mu_{\mathscr{F}_{r}}\right)+\lambda \zeta\left(\mu_{\mathscr{F}_{t}}\right)\right), \chi^{-1}\left(\lambda \chi\left(\nu_{\mathscr{F}_{r}}\right)+\lambda \chi\left(\nu_{\mathscr{F}_{t}}\right)\right)\right\rangle\right\} ; \\
\mathscr{F}_{1}^{\lambda} \otimes \mathscr{F}_{2}^{\lambda}= & \left\{\left\langle\Delta\left(\zeta^{-1}\left(\zeta\left(\zeta^{-1}\left(\lambda \zeta\left(\Delta_{\mathscr{F}_{r}}^{-1}\right)\right)+\zeta^{-1}\left(\lambda \zeta\left(\triangle_{\mathscr{F}_{t}}^{-1}\right)\right)\right)\right)\right), \zeta^{-1}\left(\zeta\left(\zeta^{-1}\left(\lambda \zeta\left(\mu_{\mathscr{F}_{r}}\right)\right)+\zeta^{-1}\left(\lambda \zeta\left(\mu_{\mathscr{F}_{t}}\right)\right)\right), \chi^{-1}\left(\chi \left(\chi^{-1}\left(\lambda \chi\left(\nu_{\mathscr{F}_{r}}\right)\right)\right.\right.\right.\right.\right. \\
& \left.\left.\left.\left.+\chi^{-1}\left(\lambda \chi\left(\nu_{\mathscr{F}_{t}}\right)\right)\right)\right)\right\rangle\right\} \\
= & \left\{\left\langle\Delta\left(\zeta^{-1}\left(\lambda \zeta\left(\Delta_{\mathscr{F}_{r}}^{-1}\right)+\lambda \zeta\left(\Delta_{\mathscr{F}_{t}}^{-1}\right)\right)\right), \zeta^{-1}\left(\lambda \zeta\left(\mu_{\mathscr{F}_{r}}\right)+\lambda \zeta\left(\mu_{\mathscr{F}_{t}}\right)\right), \chi^{-1}\left(\lambda \chi\left(\nu_{\mathscr{F}_{r}}\right)+\lambda \chi\left(\nu_{\mathscr{F}_{t}}\right)\right)\right\rangle\right\} .
\end{aligned}
$$

So $\left(\mathscr{F}_{1} \otimes \mathscr{F}_{2}\right)^{\lambda}=\mathscr{F}_{1}^{\lambda} \otimes \mathscr{F}_{2}^{\lambda}$ holds.

$$
\begin{aligned}
\lambda_{1} \mathscr{F}_{1} \oplus \lambda_{2} \mathscr{F}_{1}= & \left\{\left\langle\Delta\left(\chi^{-1}\left(\chi\left(\chi^{-1}\left[\lambda_{1} \chi\left(\Delta_{\mathscr{F}_{r}}^{-1}\right)+\lambda_{2} \chi\left(\Delta_{\mathscr{F}_{r}}^{-1}\right)\right]\right)\right)\right), \chi^{-1}\left(\chi\left(\chi^{-1}\left[\lambda_{1} \chi\left(\mu_{\mathscr{F}_{r}}\right)+\lambda_{2} \chi\left(\mu_{\mathscr{F}_{r}}\right)\right]\right)\right), \zeta^{-1}\left(\zeta \left(\zeta ^ { - 1 } \left[\lambda_{1} \zeta\left(\nu_{\mathscr{F}_{r}}\right)\right.\right.\right.\right.\right. \\
& \left.\left.\left.\left.\left.+\lambda_{2} \zeta\left(v_{\mathscr{F}_{r}}\right)\right]\right)\right)\right\rangle\right\} \\
= & \left\{\left\langle\Delta\left(\chi^{-1}\left(\left(\lambda_{1}+\lambda_{2}\right) \chi\left(\Delta_{\mathscr{F}_{r}}^{-1}\right)\right)\right), \chi^{-1}\left(\left(\lambda_{1}+\lambda_{2}\right) \chi\left(\mu_{\mathscr{F}_{r}}\right)\right), \zeta^{-1}\left(\left(\lambda_{1}+\lambda_{2}\right) \zeta\left(\nu_{\mathscr{F}_{r}}\right)\right)\right\rangle\right\} \\
= & \left(\lambda_{1}+\lambda_{2}\right) \mathscr{F}_{1} .
\end{aligned}
$$

By using the above equations, we can obtain $\left(\lambda_{1}+\lambda_{2}\right) \mathscr{F}_{1}=\lambda_{1} \mathscr{F}_{1} \oplus \lambda_{2} \mathscr{F}_{1}$. 


$$
\begin{aligned}
\mathscr{F}_{1}^{\lambda_{1} \otimes \mathscr{F}_{1}} \lambda_{2}= & \left\{\left\langle\Delta\left(\zeta^{-1}\left(\zeta\left(\zeta^{-1}\left(\lambda_{1} \zeta\left(\Delta_{\mathscr{F}_{r}}^{-1}\right)\right)+\zeta^{-1}\left(\lambda_{2} \zeta\left(\Delta_{\mathscr{F}_{r}}^{-1}\right)\right)\right)\right)\right), \zeta^{-1}\left(\zeta\left(\zeta^{-1}\left(\lambda_{1} \zeta\left(\mu_{\mathscr{F}_{r}}\right)\right)+\zeta^{-1}\left(\lambda_{2} \zeta\left(\mu_{\mathscr{F}_{r}}\right)\right)\right)\right), \chi^{-1}\left(\chi \left(\chi^{-1}\left(\lambda_{1} \chi\left(\nu_{\mathscr{F}_{r}}\right)\right)\right.\right.\right.\right. \\
& \left.\left.\left.\left.+\chi^{-1}\left(\lambda_{2} \chi\left(\nu_{\mathscr{F}_{r}}\right)\right)\right)\right)\right\rangle\right\} \\
= & \left\{\left\langle\Delta\left(\zeta^{-1}\left(\left(\lambda_{r}+\lambda_{2}\right) \zeta\left(\Delta_{\mathscr{F}_{r}}^{-1}\right)\right)\right), \zeta^{-1}\left(\left(\lambda_{1}+\lambda_{2}\right) \zeta\left(\mu_{\mathscr{F}_{r}}\right)\right), \chi^{-1}\left(\left(\lambda_{1}+\lambda_{2}\right) \chi\left(\nu_{\mathscr{F}_{r}}\right)\right)\right\rangle\right\} \\
= & \mathscr{F}_{1}^{\lambda_{1}+\lambda_{2}}
\end{aligned}
$$

\section{ATS-Based HF2TLSs Aggregation Operators}

In this section, using the operational laws of HF2TLSs defined in the previous section and extending the Bonferroni mean (BM) to the context of HF2TLSs, we introduce the related definitions of ATS-based HF2TLSs aggregation operators.

\subsection{A-HF2TLWBM Aggregation Operator}

Definition 10. Let $\left\{\mathscr{F}_{r} \mid r=1,2, \ldots, n\right\}$ be a collection of HF2TLNs, $\mathbf{w}=\left(w_{1}, w_{2}, \ldots, w_{n}\right)$ with $w_{r} \in[0,1]$ be a weight vector, and $p, q>0$ :

$$
\operatorname{A-HF} 2 \operatorname{TLWBM}^{p, q}\left(\mathscr{F}_{1}, \mathscr{F}_{2}, \ldots, \mathscr{F}_{n}\right)=\bigcup_{\mathscr{F}_{1}, \ldots, \mathscr{F}_{n}}\left(\frac{1}{n(n-1)} \stackrel{\oplus}{r}, t=1, r \neq t^{n}\left(w_{r} \mathscr{F}_{r}\right)^{p} \otimes\left(w_{t} \mathscr{F}_{t}\right)^{q}\right)^{1 /(p+q)}
$$

is called an ATS-based hesitant Fermatean 2-tuple linguistic weighted Bonferroni mean aggregation operator.
Theorem 2. Let $\left\{\mathscr{F}_{r} \mid r=1,2, \ldots, n\right\}$ be a collection of HF2TLNs, then the result derived from Definition 10 is formulated as follows $(N=1 /(n(n-1)))$ :

$$
\begin{aligned}
& \text { A-HF2TLWBM }{ }^{p, q}\left(\mathscr{F}_{1}, \mathscr{F}_{2}, \ldots, \mathscr{F}_{n}\right)=\bigcup_{\mathscr{F}_{1}, \ldots, \mathscr{F}_{n}}\left\{\left\langle\Delta\left(\zeta^{-1}\left(\frac{1}{p+q} \zeta\left(\chi^{-1}\left(N \sum_{r, t=1, r \neq t}^{n} \chi\left(\zeta^{-1}\left[p \zeta\left(\chi^{-1}\left(w_{r} \chi\left(\Delta_{\mathscr{F}_{r}}^{-1}\right)\right)\right)+q \zeta\left(\chi^{-1}\left(w_{t} \chi\left(\Delta_{\mathscr{F}_{t}}^{-1}\right)\right)\right)\right]\right)\right)\right)\right)\right),\right.\right. \\
& \zeta^{-1}\left(\frac{1}{p+q} \zeta\left(\chi^{-1}\left(N \sum_{r, t=1, r \neq t}^{n} \chi\left(\zeta^{-1}\left[p \zeta\left(\chi^{-1}\left(w_{r} \chi\left(\mu_{\mathscr{F}_{r}}\right)\right)\right)+q \zeta\left(\chi^{-1}\left(w_{t} \chi\left(\mu_{\mathscr{F}_{t}}\right)\right)\right)\right]\right)\right)\right)\right) \\
& \left.\left.\chi^{-1}\left(\frac{1}{p+q} \chi\left(\zeta^{-1}\left(N \sum_{r, t=1, r \neq t}^{n} \zeta\left(\chi^{-1}\left[p \chi\left(\zeta^{-1}\left(w_{r} \zeta\left(v_{\mathscr{F}_{r}}\right)\right)\right)+q \chi\left(\zeta^{-1}\left(w_{t} \zeta\left(v_{\mathscr{F}_{t}}\right)\right)\right)\right]\right)\right)\right)\right)\right\rangle\right\}
\end{aligned}
$$

Theorem 3. Let $\left\{\mathscr{F}_{r} \mid r=1,2, \ldots, n\right\}$ be a collection of HF2TLNs and $p, q>0$; three properties of the A-HF2TLWBM operator can be obtained as follows:

(1) Idempotency: if $\mathscr{F}_{r}=\mathscr{F}$ for all $r$, then

$$
\operatorname{A-HF2TLWBM}{ }^{p, q}\left(\mathscr{F}_{1}, \mathscr{F}_{2}, \ldots, \mathscr{F}_{n}\right)=\mathscr{F} \text {. }
$$

(2) Monotonicity: assume that $\left\langle\left(s f_{\theta\left(\mathscr{F}_{r}\right)}, \alpha_{\mathscr{F}_{F}}\right)\right.$, $\left.\mu_{\mathscr{F}_{r}}, v_{\mathscr{F}_{r}}\right\rangle \in \mathscr{F}_{r},\left\langle\left(s f_{\theta\left(\mathscr{F}^{\prime}\right)}, \alpha_{\mathscr{F}^{\prime}}\right), \mu_{\mathscr{F}_{r}^{\prime}}, v_{\mathscr{F}^{\prime}}\right\rangle \in \mathscr{F}_{r}^{\prime}$. If $\mathscr{F}_{r}$ and $\mathscr{F}_{r}^{r}$ satisfy $\left\langle\left(s f_{\theta\left(\mathscr{F}_{r}\right)}, \alpha_{\mathscr{F}_{r}}\right), \mu_{\mathscr{F}_{r}}, \nu_{\mathscr{F}_{r}}\right\rangle \leq\left\langle\left(s f_{\theta\left(\mathscr{F}_{r}^{\prime}\right)}\right.\right.$, $\left.\left.\alpha_{\mathscr{F}_{r}^{\prime}}\right), \mu_{\mathscr{F}_{r}^{\prime}}, v_{\mathscr{F}_{r}^{\prime}}\right\rangle$, then $\left.\left(s f_{\theta\left(\mathscr{F}_{r}\right)}, \alpha_{\mathscr{F}_{r}}\right) \leq\left(s f_{\theta\left(\mathscr{F}_{r}^{\prime}\right.}\right), \alpha_{\mathscr{F}_{r}^{\prime}}\right)$, $\mu_{\mathscr{F}_{r}} \leq \mu_{\mathscr{F}_{r}^{\prime}}, v_{\mathscr{F}_{r}} \geq v_{\mathscr{F}_{r}^{\prime}}:$

$$
\begin{aligned}
& \operatorname{A-HF2TLWBM}^{p, q}\left(\mathscr{F}_{1}, \mathscr{F}_{2}, \ldots, \mathscr{F}_{n}\right) \\
& \leq \operatorname{A-HF} \operatorname{TLWBM}^{p, q}\left(\mathscr{F}_{1}^{\prime}, \mathscr{F}_{2}^{\prime}, \ldots, \mathscr{F}_{n}^{\prime}\right) \text {. }
\end{aligned}
$$

(3) Boundedness: denote $\mathscr{F}^{+}=\max \left\{\mathscr{F}_{r}\right\}=\{\langle\max$ $\left.\left.\left(s f_{\theta\left(\mathscr{F}_{r}\right.}, \alpha_{\mathscr{F}_{r}}\right), \max \left(\mu_{\mathscr{F}_{r}}\right), \min \left(\nu_{\mathscr{F}_{r}}\right)\right\rangle\right\}$ and $\mathscr{F}^{-}=\min$ $\left\{\mathscr{F}_{r}\right\}=\left\{\left\langle\min \left(s f_{\theta\left(\mathscr{F}_{r}\right)}, \alpha_{\mathscr{F}_{r}}\right), \min \left(\mu_{\mathscr{F}_{r}}\right), \max \left(\nu_{\mathscr{F}_{r}}\right)\right\rangle\right\}$, then

$$
\mathscr{F}^{-} \leq \operatorname{A-HF} 2 \operatorname{TLWBM}^{p, q}\left(\mathscr{F}_{r}, \mathscr{F}_{2}, \ldots, \mathscr{F}_{n}\right) \leq \mathscr{F}^{+}
$$


Proof

$$
\begin{aligned}
& \operatorname{A-HF} \operatorname{HLWBM}^{p, q}\left(\mathscr{F}_{1}, \mathscr{F}_{2}, \ldots, \mathscr{F}_{n}\right) \Longrightarrow \mathrm{A}-\operatorname{HF}_{2} \operatorname{TLWBM}^{p, q}(\mathscr{F}, \mathscr{F}, \ldots, \mathscr{F}) \\
& =\bigcup_{\mathscr{F}_{1}, \ldots, \mathscr{F}_{n}}\left\{\left\langle\Delta\left(\zeta^{-1}\left(\frac{1}{p+q} \zeta\left(\chi^{-1}\left(N \frac{1}{N} \chi\left(\zeta^{-1}\left[p \zeta\left(\chi^{-1}\left(w \chi\left(\Delta_{\mathscr{F}}^{-1}\right)\right)\right)+q \zeta\left(\chi^{-1}\left(w \chi\left(\Delta_{\mathscr{F}}^{-1}\right)\right)\right)\right]\right)\right)\right)\right)\right),\right.\right. \\
& \zeta^{-1}\left(\frac{1}{p+q} \zeta\left(\chi^{-1}\left(\chi\left(\zeta^{-1}\left[p \zeta\left(\chi^{-1}\left(w \chi\left(\mu_{\mathscr{F}}\right)\right)\right)+q \zeta\left(\chi^{-1}\left(w \chi\left(\mu_{\mathscr{F}}\right)\right)\right)\right]\right)\right)\right)\right) \\
& \left.\left.\chi^{-1}\left(\frac{1}{p+q} \chi\left(\zeta^{-1}\left(\zeta\left(\chi^{-1}\left[p \zeta\left(\chi^{-1}\left(w \zeta\left(\nu_{\mathscr{F}}\right)\right)\right)+q \chi\left(\zeta^{-1}\left(w \zeta\left(\nu_{\mathscr{F}}\right)\right)\right)\right]\right)\right)\right)\right)\right\rangle\right\} \\
& =\underset{\mathscr{F}, \ldots, \mathscr{F}}{\cup}\left\{\left\langle\Delta\left(\zeta^{-1}\left(\frac{1}{p+q} \zeta\left(\zeta^{-1}\left[(p+q) \zeta\left(\chi^{-1}\left(w \chi\left(\Delta_{\mathscr{F}}^{-1}\right)\right)\right)\right]\right)\right)\right)\right.\right. \\
& \zeta^{-1}\left(\frac{1}{p+q} \zeta\left(\zeta^{-1}\left[(p+q) \zeta\left(\chi^{-1}\left(w \chi\left(\mu_{\mathscr{F}}\right)\right)\right)\right]\right)\right) \\
& \left.\left.\chi^{-1}\left(\frac{1}{p+q} \chi\left(\chi^{-1}\left[(p+q) \chi\left(\zeta^{-1}\left(w \zeta\left(\nu_{\mathscr{F}}\right)\right)\right)\right]\right)\right)\right\rangle\right\} \\
& =\mathscr{F} \text {. }
\end{aligned}
$$

$\zeta$ and $\zeta^{-1}$ are strictly decreasing functions; $\chi$ and $\chi^{-1}$ are strictly increasing functions. From Theorem 2 , we can obtain that

$$
\begin{aligned}
& \operatorname{A-HF} 2 \operatorname{TLWBM}{ }^{p, q}\left(\mathscr{F}_{1}, \mathscr{F}_{2}, \ldots, \mathscr{F}_{n}\right)=\bigcup_{\mathscr{F}_{1}, \ldots, \mathscr{F}_{n}}\left\{\left\langle\Delta\left(\zeta^{-1}\left(\frac{1}{p+q} \zeta\left(\chi^{-1}\left(N \sum_{r, t=1, r \neq t}^{n} \chi\left(\zeta^{-1}\left[p \zeta\left(\chi^{-1}\left(w_{r} \chi\left(\Delta_{\mathscr{F}_{r}}^{-1}\right)\right)\right)+q \zeta\left(\chi^{-1}\left(w_{t} \chi\left(\Delta_{\mathscr{F}_{t}}^{-1}\right)\right)\right)\right]\right)\right)\right)\right)\right)\right.\right. \\
& \zeta^{-1}\left(\frac{1}{p+q} \zeta\left(\chi^{-1}\left(N \sum_{r, t=1, r \neq t}^{n} \chi\left(\zeta^{-1}\left[p \zeta\left(\chi^{-1}\left(w_{r} \chi\left(\mu_{\mathscr{F}_{r}}\right)\right)\right)+q \zeta\left(\chi^{-1}\left(w_{t} \chi\left(\mu_{\mathscr{F}_{t}}\right)\right)\right)\right]\right)\right)\right)\right) \\
& \left.\left.\chi^{-1}\left(\frac{1}{p+q} \chi\left(\zeta^{-1}\left(N \sum_{r, t=1, r \neq t}^{n} \zeta\left(\chi^{-1}\left[p \chi\left(\zeta^{-1}\left(w_{r} \zeta\left(v_{\mathscr{F}_{r}}\right)\right)\right)+q \chi\left(\zeta^{-1}\left(w_{t} \zeta\left(v_{\mathscr{F}_{t}}\right)\right)\right)\right]\right)\right)\right)\right)\right\rangle\right\} \\
& \leq \underset{\mathscr{F}_{1}^{\prime}, \ldots, \mathscr{F}_{n}^{\prime}}{\bigcup}\left\{\left\langle\Delta\left(\zeta^{-1}\left(\frac{1}{p+q} \zeta\left(\chi^{-1}\left(N \times \sum_{r, t=1, r \neq t}^{n} \chi\left(\zeta^{-1}\left[p \zeta\left(\chi^{-1}\left(w_{r}^{\prime} \chi\left(\Delta_{\mathscr{F}_{r}^{\prime}}^{-1}\right)\right)\right)+q \zeta\left(\chi^{-1}\left(w_{t}^{\prime} \chi\left(\Delta_{\mathscr{F}_{t}^{\prime}}^{-1}\right)\right)\right)\right]\right)\right)\right)\right)\right),\right.\right. \\
& \zeta^{-1}\left(\frac{1}{p+q} \zeta\left(\chi^{-1}\left(N \sum_{r, t=1, r \neq t}^{n} \chi\left(\zeta^{-1}\left[p \zeta\left(\chi^{-1}\left(w_{r}^{\prime} \chi\left(\mu_{\mathscr{F}_{r}^{\prime}}\right)\right)\right)+q \zeta\left(\chi^{-1}\left(w_{t}^{\prime} \chi\left(\mu_{\mathscr{F}_{t}^{\prime}}\right)\right)\right)\right]\right)\right)\right)\right) \\
& \left.\chi^{-1}\left(\frac{1}{p+q} \chi\left(\zeta^{-1}\left(N \sum_{r, t=1, r \neq t}^{n} \zeta\left(\chi^{-1}\left[p \chi\left(\zeta^{-1}\left(w_{r}^{\prime} \zeta\left(\nu_{\mathscr{F}_{r}^{\prime}}\right)\right)\right)+q \chi\left(\zeta^{-1}\left(w_{t}^{\prime} \zeta\left(\nu_{\mathscr{F}_{t}^{\prime}}\right)\right)\right)\right]\right)\right)\right)\right)\right\rangle, \\
& =\mathrm{A}-\mathrm{HF}_{2} \operatorname{TLWBM}^{p, q}\left(\mathscr{F}_{1}^{\prime}, \mathscr{F}_{2}^{\prime}, \ldots, \mathscr{F}_{n}^{\prime}\right) \text {, }
\end{aligned}
$$


where $\left\langle\left(s f_{\theta\left(\mathscr{F}_{r}\right)}, \alpha_{\mathscr{F}_{r}}\right), \mu_{\mathscr{F}_{r}}, v_{\mathscr{F}_{r}}\right\rangle \in \mathscr{F}_{r}, \Delta_{\mathscr{F}_{r}}^{-1}=\Delta^{-1}\left(s f_{\theta\left(\mathscr{F}_{r}\right)}\right)$ $\left.\alpha_{\mathscr{F}_{r}}\right), \quad\left\langle\left(s f_{\theta\left(\mathscr{F}_{r}^{\prime}\right)}, \alpha_{\mathscr{F}_{r}^{\prime}}\right), \mu_{\mathscr{F}_{r}^{\prime}}, v_{\mathscr{F}_{r}^{\prime}}\right\rangle \in \mathscr{F}_{r}^{\prime}, \Delta_{\mathscr{F}_{r}^{\prime}}^{-1}=\Delta^{-1}\left(s f_{\theta\left(\mathscr{F}_{r}^{\prime}\right.}\right)$, $\left.\alpha_{\mathscr{F}_{r}^{\prime}}\right)$, and $N=1 /(n(n-1))$.

According to the idempotency property, we obtain A-HF2TLWBM ${ }^{p, q}\left(\mathscr{F}_{1}^{+}, \mathscr{F}_{2}^{+}, \ldots, \mathscr{F}_{n}^{+}\right)=\mathscr{F}^{+}$and A-HF2TLWBM ${ }^{p, q}\left(\mathscr{F}_{1}^{-}, \mathscr{F}_{2}^{-}, \ldots, \mathscr{F}_{n}^{-}\right)=\mathscr{F}^{-}$. Using the monotonicity, we obtain the following inequalities:
A-HF2TLWBM ${ }^{p, q}\left(\mathscr{F}_{1}^{-}, \mathscr{F}_{2}^{-}, \ldots, \mathscr{F}_{n}^{-}\right) \leq \mathrm{A}-\mathrm{HF} 2$ $\operatorname{TLWBM}^{p, q}\left(\mathscr{F}_{1}, \mathscr{F}_{2}, \ldots, \mathscr{F}_{n}\right) \leq \mathrm{A}-\mathscr{H F}_{2} \operatorname{TLWBM}^{p, q}\left(\mathscr{F}_{1}^{+}\right.$, $\left.\mathscr{F}_{2}^{+}, \ldots, \mathscr{F}_{n}^{+}\right)$. Thus, $\mathscr{F}^{-} \leq \mathrm{A}-\operatorname{HF}_{2} \operatorname{TLWBM}^{p, q}\left(\mathscr{F}_{1}, \mathscr{F}_{2}\right.$, $\left.\ldots, \mathscr{F}_{n}\right) \leq \mathscr{F}^{+}$.

Furthermore, some special cases are examined by taking different parameters values $(N=1 /(n(n-1)))$.

Case 1 . When $q \longrightarrow 0$, then we have

$$
\begin{aligned}
\operatorname{A-HF2TLWBM}^{p, 0}\left(\mathscr{F}_{1}, \mathscr{F}_{2}, \ldots, \mathscr{F}_{n}\right)= & \bigcup_{\mathscr{F}_{1}, \ldots, \mathscr{F}_{n}}\left(N \bigoplus_{r=1}^{n}\left(w_{r} \mathscr{F}_{r}\right)^{p}\right)^{1 / p} \\
= & \bigcup_{\mathscr{F}_{1}, \ldots, \mathscr{F}_{n}}\left\{\left\langle\Delta\left(\zeta^{-1}\left(\frac{1}{p} \zeta\left(\chi^{-1}\left(N \sum_{r=1}^{n} \chi\left(\zeta^{-1}\left(p \zeta\left(\chi^{-1}\left(w_{r} \chi\left(\Delta_{\mathscr{F}_{r}}^{-1}\right)\right)\right)\right)\right)\right)\right)\right)\right),\right.\right. \\
& \zeta^{-1}\left(\frac{1}{p} \zeta\left(\chi^{-1}\left(N \sum_{r=1}^{n} \chi\left(\zeta^{-1}\left(p \zeta\left(\chi^{-1}\left(w_{r} \chi\left(\mu_{\mathscr{F}_{r}}\right)\right)\right)\right)\right)\right)\right)\right), \\
& \left.\left.\chi^{-1}\left(\frac{1}{p} \chi\left(\zeta^{-1}\left(N \sum_{r=1}^{n} \zeta\left(\chi^{-1}\left(p \chi\left(\zeta^{-1}\left(w_{r} \zeta\left(\nu_{\mathscr{F}_{r}}\right)\right)\right)\right)\right)\right)\right)\right)\right\rangle\right\} .
\end{aligned}
$$

Case 2. When $p=1, q \longrightarrow 0$, then we obtain ATS-based hesitant Fermatean 2-tuple linguistic unit weighted Bonferroni mean aggregation operator as follows:

$$
\begin{aligned}
& \operatorname{A-HF2TLWBM}{ }^{1,0}\left(\mathscr{F}_{1}, \mathscr{F}_{2}, \ldots, \mathscr{F}_{n}\right)=\underset{\mathscr{F}_{1}, \ldots, \mathscr{F}_{n}}{\bigcup}\left(N \underset{r=1}{\oplus}\left(w_{r} \mathscr{F}_{r}\right)\right) \\
& \quad=\bigcup_{\mathscr{F}_{1}, \ldots, \mathscr{F}_{n}}\left\{\left\langle\Delta\left(\chi^{-1}\left(N \sum_{r=1}^{n}\left(w_{r} \chi\left(\Delta_{\mathscr{F}_{r}}^{-1}\right)\right)\right)\right), \chi^{-1}\left(N \sum_{r=1}^{n}\left(w_{r} \chi\left(\mu_{\mathscr{F}_{r}}\right)\right)\right), \zeta^{-1}\left(N \sum_{r=1}^{n}\left(w_{r} \zeta\left(\nu_{\mathscr{F}_{r}}\right)\right)\right)\right\rangle\right\} .
\end{aligned}
$$

Case 3. When $p=1, q=1$, then we get ATS-based hesitant Fermatean 2-tuple linguistic interrelated square weighted Bonferroni mean aggregation operator as follows:

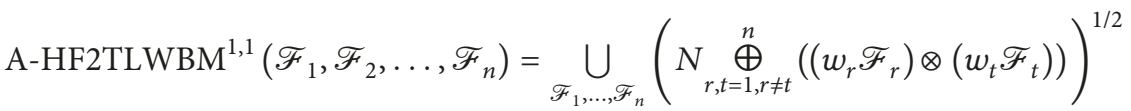

$$
\begin{aligned}
& ={\underset{\mathscr{F}}{1}, \ldots, \mathscr{F}_{n}}_{U}\left\{\left\langle\Delta\left(\zeta^{-1}\left(\frac{1}{2} \zeta\left(\chi^{-1}\left(N \sum_{r, t=1, r \neq t}^{n}\left(w_{r} \chi\left(\Delta_{\mathscr{F}_{r}}^{-1}\right)\right)+\left(w_{t} \chi\left(\Delta_{\mathscr{F}_{t}}^{-1}\right)\right)\right)\right)\right)\right),\right.\right. \\
& \zeta^{-1}\left(\frac{1}{2} \zeta\left(\chi^{-1}\left(N \sum_{r, t=1, r \neq t}^{n}\left(w_{r} \chi\left(\mu_{\mathscr{F}_{r}}\right)\right)+\left(w_{t} \chi\left(\mu_{\mathscr{F}_{t}}\right)\right)\right)\right)\right) \\
& \left.\left.\chi^{-1}\left(\frac{1}{2} \chi\left(\zeta^{-1}\left(N \sum_{r, t=1, r \neq t}^{n}\left(w_{r} \zeta\left(\nu_{\mathscr{F}_{r}}\right)\right)+\left(w_{t} \zeta\left(\nu_{\mathscr{F}_{t}}\right)\right)\right)\right)\right)\right\rangle\right\} .
\end{aligned}
$$


4.2. A-HF2TLWGBM Aggregation Operator. In the sequel, the classical GBM operator is extended under the background of HF2TLSs situation, and we derive the following aggregation operator.
Definition 11. Let $\left\{\mathscr{F}_{r} \mid r=1,2, \ldots, n\right\}$ be a collection of HF2TLNs, $\mathbf{w}=\left(w_{1}, w_{2}, \ldots, w_{n}\right)$ be a weight vector, and $p, q>0$; then,

$$
\operatorname{A-HF} 2 \operatorname{TLWGBM}{ }^{p, q}\left(\mathscr{F}_{1}, \mathscr{F}_{2}, \ldots, \mathscr{F}_{n}\right)=\underset{\mathscr{F}_{1}, \ldots, \mathscr{F}_{n}}{\cup} \frac{1}{p+q}\left(\bigotimes_{r, t=1, r \neq t}^{n}\left(p \mathscr{F}_{r}^{w_{r}} \oplus q \mathscr{F}_{t}^{w_{t}}\right)^{1 /(n(n-1))}\right)
$$

which is called an ATS-based hesitant Fermatean 2-tuple linguistic weighted geometric Bonferroni mean.
Theorem 4. Let $\left\{\mathscr{F}_{r} \mid r=1,2, \ldots, n\right\}$ be a collection of HF2TLNs; then, the result derived from Definition 11 is also a HF2TLS and $(N=1 /(n(n-1)))$ :

$$
\begin{aligned}
\operatorname{A-HF} \operatorname{TLWGBM}^{p, q}\left(\mathscr{F}_{1}, \mathscr{F}_{2}, \ldots, \mathscr{F}_{n}\right)= & \bigcup_{\mathscr{F}_{1}, \ldots, \mathscr{F}_{n}} \\
& \cdot\left\{\left\langle\Delta\left(\chi^{-1}\left(\frac{1}{p+q} \chi\left(\zeta^{-1}\left(\sum_{r, t=1, r \neq t}^{n} N \zeta\left(\chi^{-1}\left(p \chi\left(\zeta^{-1}\left(w_{r} \zeta\left(\Delta_{\mathscr{F}_{r}}^{-1}\right)\right)\right)+q \chi\left(\zeta^{-1}\left(w_{t} \zeta\left(\Delta_{\mathscr{F}_{t}}^{-1}\right)\right)\right)\right)\right)\right)\right)\right)\right),\right.\right. \\
& \chi^{-1}\left(\frac{1}{p+q} \chi\left(\zeta^{-1}\left(\sum_{r, t=1, r \neq t}^{n} N \zeta\left(\chi^{-1}\left(p \chi\left(\zeta^{-1}\left(w_{r} \zeta\left(\mu_{\mathscr{F}_{r}}\right)\right)\right)+q \chi\left(\zeta^{-1}\left(w_{t} \zeta\left(\mu_{\mathscr{F}_{t}}\right)\right)\right)\right)\right)\right)\right)\right), \\
& \left.\left.\zeta^{-1}\left(\frac{1}{p+q} \zeta\left(\chi^{-1}\left(\sum_{r, t=1, r \neq t}^{n} N \chi\left(\zeta^{-1}\left(p \zeta\left(\chi^{-1}\left(w_{r} \chi\left(\nu_{\mathscr{F}_{r}}\right)\right)\right)+q \zeta\left(\chi^{-1}\left(w_{t} \chi\left(\nu_{\mathscr{F}_{t}}\right)\right)\right)\right)\right)\right)\right)\right)\right)\right\} .
\end{aligned}
$$

Similar to the A-HF2TLWBM operator, we can obtain that the A-HF2TLWGBM operator has also idempotency, boundedness, and monotonicity. In addition, some special forms of the A-HF2TLWGBM operator can be given according to the changes of parameters $p$ and $q$ as follows.

Case 1 . When $q \longrightarrow 0$, then the following equality holds:

$$
\begin{aligned}
\operatorname{A-HF} \operatorname{TLWGBM}^{p, 0}\left(\mathscr{F}_{1}, \mathscr{F}_{2}, \ldots, \mathscr{F}_{n}\right)= & \underset{\mathscr{F}_{1}, \ldots, \mathscr{F}_{n}}{\cup} \frac{1}{p}\left(\bigoplus_{r=1}^{n}\left(p \mathscr{F}_{r}^{w_{r}}\right)^{N}\right) \\
= & \bigcup_{\mathscr{F}_{1}, \ldots, \mathscr{F}_{n}}\left\{\left\langle\Delta\left(\chi^{-1}\left(\frac{1}{p^{\prime}} \chi\left(\zeta^{-1}\left(\sum_{r=1}^{n} N \zeta\left(\chi^{-1}\left(p \chi\left(\zeta^{-1}\left(w_{r} \zeta\left(\triangle_{\mathscr{F}_{r}}^{-1}\right)\right)\right)\right)\right)\right)\right)\right)\right)\right.\right. \\
& \chi^{-1}\left(\frac{1}{p} \chi\left(\zeta^{-1}\left(\sum_{r=1}^{n} N \zeta\left(\chi^{-1}\left(p \chi\left(\zeta^{-1}\left(w_{r} \zeta\left(\mu_{\mathscr{F}_{r}}\right)\right)\right)\right)\right)\right)\right)\right) \\
& \left.\left.\zeta^{-1}\left(\frac{1}{p} \zeta\left(\chi^{-1}\left(\sum_{r=1}^{n} N \chi\left(\zeta^{-1}\left(p \zeta\left(\chi^{-1}\left(w_{r} \chi\left(\nu_{\mathscr{F}_{r}}\right)\right)\right)\right)\right)\right)\right)\right)\right\rangle\right\}
\end{aligned}
$$


Case 2. When $p=1$ and $q \longrightarrow 0$, then the ATS-based hesitant Fermatean 2-tuple linguistic weighted unit geometric Bonferroni mean operator holds:

$$
\begin{aligned}
& \operatorname{A-HF2TLWGBM}{ }^{1,0}\left(\mathscr{F}_{1}, \mathscr{F}_{2}, \ldots, \mathscr{F}_{n}\right)={\underset{\mathscr{F}}{1}, \ldots, \mathscr{F}_{n}}_{r=1}\left(\bigoplus_{r}^{n}\left(\mathscr{F}_{r}^{w_{r}}\right)^{N}\right), \\
& ={\underset{\mathscr{F}}{1}, \ldots, \mathscr{F}_{n}}_{\bigcup}\left\{\left\langle\Delta\left(\zeta^{-1}\left(\sum_{r=1}^{n} N w_{r} \zeta\left(\Delta_{\mathscr{F}_{r}}^{-1}\right)\right)\right), \zeta^{-1}\left(\sum_{r=1}^{n} N w_{r} \zeta\left(\mu_{\mathscr{F}_{r}}\right)\right), \chi^{-1}\left(\sum_{r=1}^{n} N w_{r} \chi\left(\nu_{\mathscr{F}_{r}}\right)\right)\right\rangle\right\} .
\end{aligned}
$$

Case 3. When $p=1$ and $q=1$, then we obtain the ATSbased hesitant Fermatean 2-tuple linguistic weighted interrelated square geometric Bonferroni mean operator:

$$
\begin{aligned}
& \operatorname{A-HF} 2 \operatorname{TLWGBM}{ }^{1,1}\left(\mathscr{F}_{1}, \mathscr{F}_{2}, \ldots, \mathscr{F}_{n}\right)=\bigcup_{\mathscr{F}_{1}, \ldots, \mathscr{F}_{n}} \frac{1}{2}\left(\stackrel{\bigotimes}{\otimes}_{r, t=1, r \neq t}^{n}\left(\mathscr{F}_{r}^{w_{r}} \oplus \mathscr{F}_{t}^{w_{t}}\right)^{N}\right) \\
& =\bigcup_{\mathscr{F}_{1}, \ldots, \mathscr{F}_{n}}\left\{\left\langle\Delta\left(\chi^{-1}\left(\frac{1}{2} \chi\left(\zeta^{-1}\left(\sum_{r, t=1, r \neq t}^{n} N \zeta\left(\chi^{-1}\left(\chi\left(\zeta^{-1}\left(w_{r} \zeta\left(\triangle_{\mathscr{F}_{r}}^{-1}\right)\right)\right)+\chi\left(\zeta^{-1}\left(w_{t} \zeta\left(\triangle_{\mathscr{F}_{t}}^{-1}\right)\right)\right)\right)\right)\right)\right)\right)\right),\right.\right. \\
& \chi^{-1}\left(\frac{1}{2} \chi\left(\zeta^{-1}\left(\sum_{r, t=1, r \neq t}^{n} N \zeta\left(\chi^{-1}\left(\chi\left(\zeta^{-1}\left(w_{r} \zeta\left(\mu_{\mathscr{F}_{r}}\right)\right)\right)+\chi\left(\zeta^{-1}\left(w_{t} \zeta\left(\mu_{\mathscr{F}_{t}}\right)\right)\right)\right)\right)\right)\right)\right) \\
& \left.\left.\zeta^{-1}\left(\frac{1}{2} \zeta\left(\chi^{-1}\left(\sum_{r, t=1, r \neq t}^{n} N \chi\left(\zeta^{-1}\left(\zeta\left(\chi^{-1}\left(w_{r} \chi\left(v_{\mathscr{F}_{r}}\right)\right)\right)+\zeta\left(\chi^{-1}\left(w_{t} \chi\left(v_{\mathscr{F}_{t}}\right)\right)\right)\right)\right)\right)\right)\right)\right\rangle\right\}
\end{aligned}
$$

\section{MCDM Approach}

We aim at applying the developed A-HF2TLWBM operator and A-HF2TLWGBM operator to solve MCDM problems with hesitant Fermatean 2-tuple linguistic information. Suppose $A=\left\{A_{1}, A_{2}, \ldots, A_{m}\right\}$ denotes a collection of $m$ alternatives and $C=\left\{C_{1}, C_{2}, \ldots, C_{n}\right\}$ denotes a set of $n$ criteria, and its corresponding weight vector is $\mathbf{w}=\left(w_{1}, w_{2}, \ldots, w_{n}\right)$, where $w_{t} \in[0,1]$ is the weight of $C_{t}$ and $\sum_{t=1}^{n} w_{t}=1$. Suppose that $H=\left(\mathscr{F}_{r t}\right)_{m \times n}$ is a hesitant Fermatean 2-tuple linguistic decision matrix, in which $\mathscr{F}_{r t}$ is an HF2TLN that indicates the assessment values of $A_{r}$ with regard to $C_{t}$. Next, we employ the proposed operators to solve the MCDM problems involving hesitant Fermatean 2tuple linguistic information. The steps are summarized as follows:

Step 1. Obtain the decision matrices $H=\left(\mathscr{F}_{r t}\right)_{m \times n}$, where $\mathscr{F}_{r t}$ represents the decision-maker's preferences.

Step 2. Normalize the decision information matrices H. For ease of comparison and information aggregation, the hesitant Fermatean 2-tuple linguistic decision information matrix $H=\left(\mathscr{F}_{r t}\right)_{m \times n}$ should be transformed the normalized matrix expressed by $\bar{H}=\left(\overline{\mathscr{F}_{r t}}\right)_{m \times n}:$

$$
\overline{\mathscr{F}}_{r t}= \begin{cases}\mathscr{F}_{r t}, & \text { benefit criteria, } \\ \left(\min _{r} \mathscr{F}_{r t}\right) \oslash \mathscr{F}_{r t}, & \text { cost criteria, }\end{cases}
$$

where $\quad\left(\min _{r} \mathscr{F}_{r t}\right) \oslash \mathscr{F}_{r t}=\left\langle\left(\Delta\left(\Delta^{-1}\left(\min _{r}\left(s f_{\theta\left(\mathscr{F}_{r t}\right)}\right.\right.\right.\right.\right.$, $\left.\left.\left.\left.\alpha_{\mathscr{F}_{r t}}\right)\right) / \Delta^{-1}\left(s f_{\theta\left(\mathscr{F}_{r t}\right)}, \alpha_{\mathscr{F}_{r t}}\right)\right)\right),\left(\min _{r} \mu_{\mathscr{F}_{r t}} / \mu_{\mathscr{F}_{r t}}\right)$, $\sqrt[3]{\left.\left(\left(\max _{r} \nu_{\mathscr{F}_{r t}}\right)^{3}-\nu_{\mathscr{F}_{r t}}^{3} / 1-\nu_{\mathscr{F}_{r t}}^{3}\right)\right\rangle}:$

$\min \mathscr{F}_{i j}=\left\langle\min _{i}\left(s_{\theta\left(\mathscr{F}_{i j}\right)}, \alpha_{\mathscr{F}_{i j}}\right), \min _{i} \mu_{\mathscr{F}_{i j}}, \max _{i} \nu_{\mathscr{F}_{i j}}\right\rangle$.

Step 3. Utilize the A-HF2TLWBM operator or the AHF2TLWGBM operator to get the overall preference values $\mathscr{F}_{r}, r=1,2, \ldots, m$ corresponding to alternative $A_{r}$. In Theorems 2 and 4 , we take $\zeta(c)=-\log c$ and $\chi(c)=-\log (1-c)$ in A-HF2TLWBM operator and AHF2TLWGBM operator. 
Step 4. Rank all the alternatives $\left\{A_{1}, A_{2}, \ldots, A_{m}\right\}$ according to Definitions 6-8 and identify the best one(s).

\section{Numerical Example}

Because of the uncertainty among companies, it is difficult for experts to use just one linguistic term with a single symbolic translation parameter to provide their preferences. To facilitate the elicitation of their preferences, they can use hesitant Fermatean fuzzy 2-tuple linguistic terms, which are more akin to human beings' cognitive processes.

6.1. Background. In this part, we study an example to demonstrate the applicability of the proposed approach in solving a practical MCDM problem with hesitant Fermatean 2-tuple linguistic information. Let us assume that an investment company wants to invest a sum of money to the optimal industry. There are four parallel alternatives $\left\{A_{1}, A_{2}, A_{3}, A_{4}\right\}$, which are a car industry, a food company, a computer company, and an arms industry, respectively. Suppose that a company committee composed of professional experts must select the best industry in accordance with the following four criteria: $C_{1}$ is the mobility analysis; $C_{2}$ is the risk analysis; $C_{3}$ is the feasibility of the project analysis; $C_{4}$ is the profitability analysis; and their weight vector is $\mathbf{w}=(0.2,0.1,0.3,0.4)$. The experts evaluate four companies by virtue of the linguistic term set $s f=\left\{s f_{0}=\right.$ extremely poor, $s f_{1}=$ poor, $s f_{2}=$ medium,

$s f_{3}=$ good, $s f_{4}=$ extremely good $\}$.

6.2. Illustration of the Proposed Method. Next, we employ an approach designed in Section 5 to identify the optimal alternative(s).

Step 1. Obtain the decision matrices $H=\left(\mathscr{F}_{r t}\right)_{m \times n}$, which are shown in Table 1 . Considering all criteria are beneficial types; the matrix $H=\left(\mathscr{F}_{r t}\right)_{m \times n}$ maintains its original form.

Step 2. Use the A-HF2TLWBM operator to utilize all preference values of the alternative $A_{r}(r=1,2,3,4)$ with $p=1, q=1, \zeta(c)=-\log c$, and $\chi(c)=-\log (1-c)$, the results are listed as follows:

$$
\begin{aligned}
& \mathscr{F}_{1}=\left\{\left\langle\left(s f_{1}, 0.0053\right), 0.4942,0.0086\right\rangle,\left\langle\left(s f_{1}, 0.0034\right), 0.5409,0.0107\right\rangle\right\}, \\
& \mathscr{F}_{2}=\left\{\left\langle\left(s f_{1}, 0.0012\right), 0.4885,0.0060\right\rangle,\left\langle\left(s f_{1}, 0.0103\right), 0.5118,0.0071\right\rangle\right\}, \\
& \mathscr{F}_{3}=\left\{\left\langle\left(s f_{1}, 0.0108\right), 0.5192,0.0064\right\rangle,\left\langle\left(s f_{1}, 0.0023\right), 0.5946,0.0097\right\rangle\right\}, \\
& \mathscr{F}_{4}=\left\{\left\langle\left(s f_{1}, 0.0027\right), 0.5016,0.0077\right\rangle,\left\langle\left(s f_{1}, 0.0093\right), 0.6287,0.0072\right\rangle,\left\langle\left(s f_{1}, 0.0101\right), 0.4616,0.0059\right\rangle,\left\langle\left(s f_{1}, 0.0073\right), 0.6035,0.0055\right\rangle\right\} .
\end{aligned}
$$

Step 3. From Definition 6, the values of score function $\operatorname{SC}\left(\mathscr{F}_{r}\right), r=1,2,3,4$ are shown as follows:

$$
\begin{aligned}
\operatorname{SC}\left(\mathscr{F}_{1}\right) & =0.1401, \\
\operatorname{SC}\left(\mathscr{F}_{2}\right) & =0.1261, \\
\operatorname{SC}\left(\mathscr{F}_{3}\right) & =0.1761, \\
\operatorname{SC}\left(\mathscr{F}_{4}\right) & =0.1745 .
\end{aligned}
$$

Step 4. According to Definition 8, the ranking is obtained as follows:

$$
A_{3}>A_{4}>A_{1}>A_{2} \text {. }
$$

So, $A_{3}$ is identified as the optimal alternative. Therefore, the best company for the investment is $A_{3}$.

6.3. Influence Discussion of Parameters $p$ and $q$. To examine the influence of parameters $p$ and $q$ in the A-HF2TLWBM operator, the changes of score values with parameters $p$ and $q$ increasing are shown in Figures 1-4, where the score values $\mathrm{SC}\left(\mathscr{F}_{r}\right)$ are shown as $p$ and $q$ changing from 0 to 3 .
Figure 5 shows $\mathrm{SC}\left(\mathscr{F}_{r}\right)$ and rankings of the alternatives when $q$ is considered as the independent variable. As we observe from Figure 5, when $q \in(0,0.1225]$, the ranking result is $A_{4}>A_{3}>A_{1}>A_{2}$, and the optimal alternative is $A_{4}$, while with $q \in(0.1225,3)$, the ranking is $A_{3}>A_{4}>A_{1}>A_{2}$, and the optimal alternative is $A_{3}$. Figures $1-5$ reflect the changes of the ranking of the alternatives with different decision-makers' investment preference risk.

Furthermore, different score function values and ranking results are calculated with the changes of $p$ and $q$ in the A-HF2TLWGBM operator in Step 3. Figures 6-9 illustrate the score values $\mathrm{SC}\left(\mathscr{F}_{r}\right)$ obtained using the AHF2TLWGBM operator as $p$ and $q$ increase from 0 to 3 . Figure 10 shows that different score values $\mathrm{SC}\left(\mathscr{F}_{r}\right)$ and ranking results with a fixed value of $p$. From Figure 10, the best alternative is $A_{4}$ when $q \in(0,0.1845]$; there are different ranking of the alternatives, and $A_{1}$ is the best alternative when $q \in(0.1845,2.6145]$, while the best alternative becomes $A_{3}$ when $q \in(2.6145,3)$. Through the above analysis, we see that the ranking result varies more as parameter $q$ changes.

From the view point of Liu et al. [27], it is worth noting that the score values $\mathrm{SC}\left(\mathscr{F}_{r}\right)$ are smaller as $p$ and $q$ increase from 0 to 3 in the A-HF2TLWBM operator than those in the A-HF2TLWGBM operator. Figures 5 and 10 indicate that 


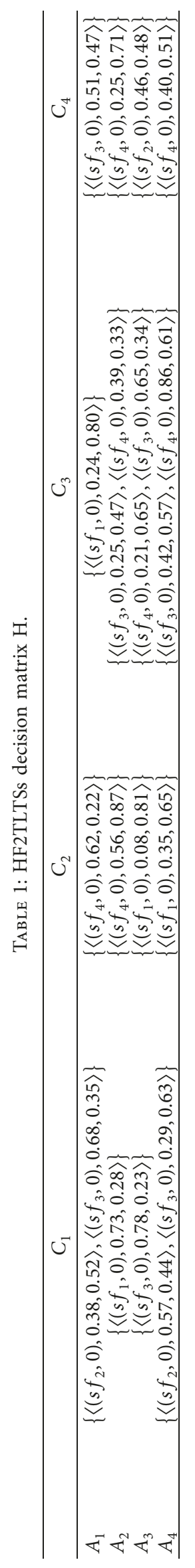




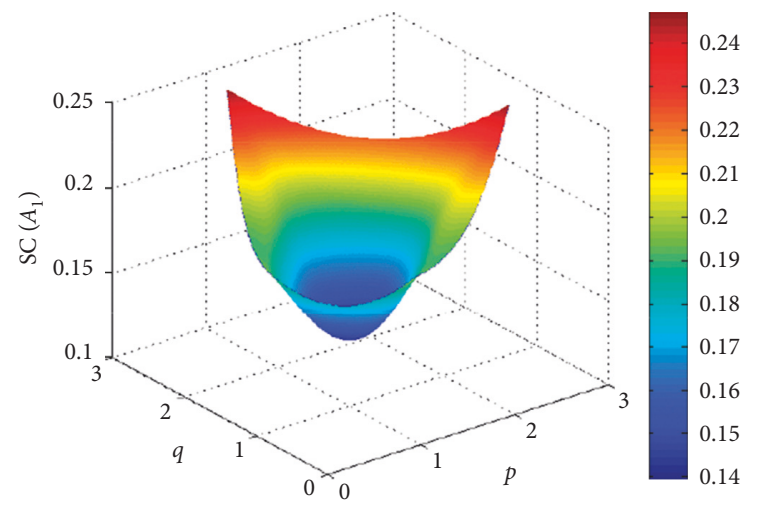

Figure 1: SC $\left(\mathscr{F}_{1}\right)$ of $A_{1}$ based on the A-HF2TLWBM operator.

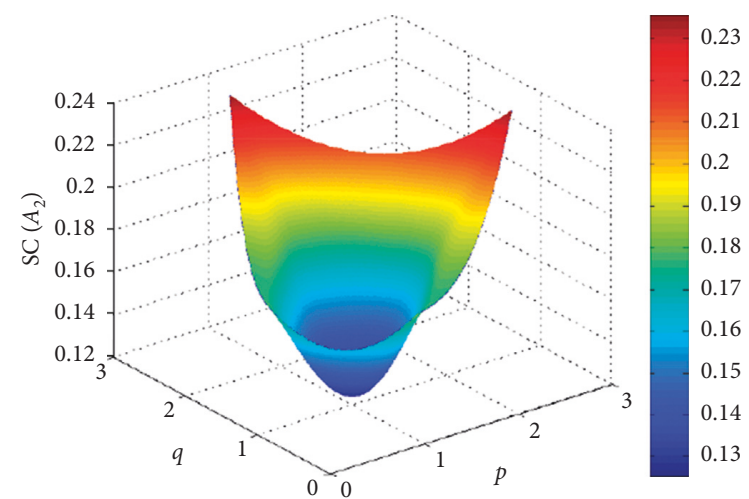

Figure 2: $\operatorname{SC}\left(\mathscr{F}_{2}\right)$ of $A_{2}$ based on the A-HF2TLWBM operator.

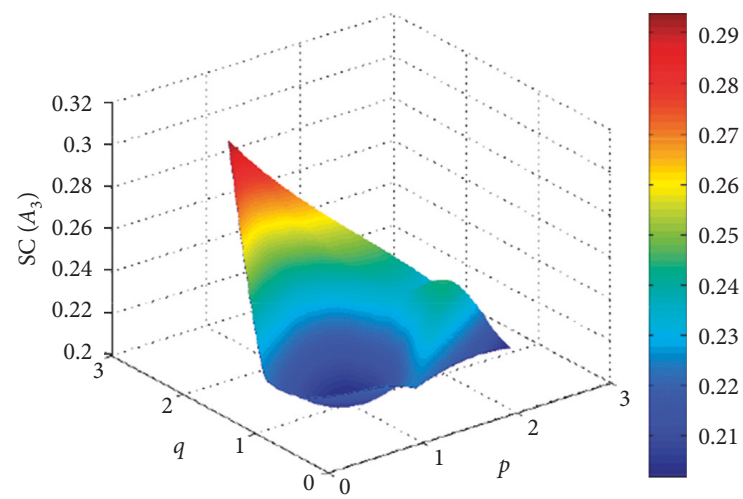

Figure 3: $\operatorname{SC}\left(\mathscr{F}_{3}\right)$ of $A_{3}$ based on the A-HF2TLWBM operator.

the A-HF2TLWBM operator may cause more disadvantageous (or pessimistic) preferences and that the AHF2TLWGBM operator possesses more advantageous (or optimistic) preferences. Hence, the A-HF2TLWBM operator can be regarded as the pessimistic operator and the AHF2TLWGBM operator as the optimistic operator, while parameters $p$ and $q$ can be considered as pessimistic or optimistic preference levels. To some extent, it can be concluded that a decision-maker delivering a pessimistic assessment of the prospect prefers to select the

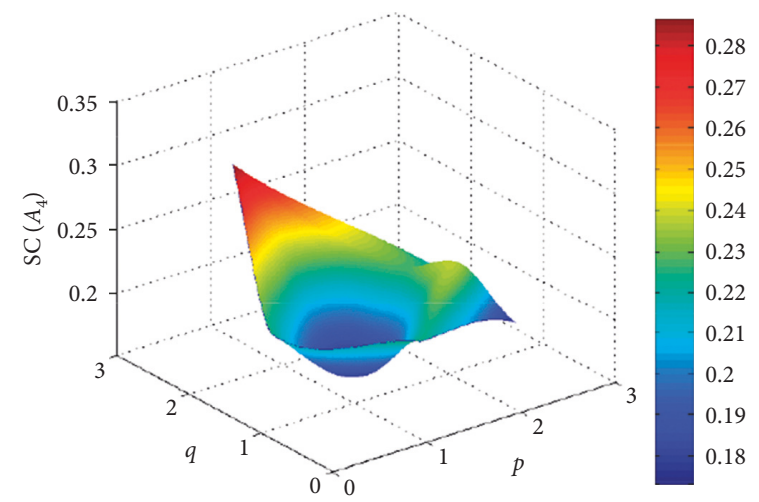

FIgURE 4: SC $\left(\mathscr{F}_{4}\right)$ of $A_{4}$ based on the A-HF2TLWBM operator.

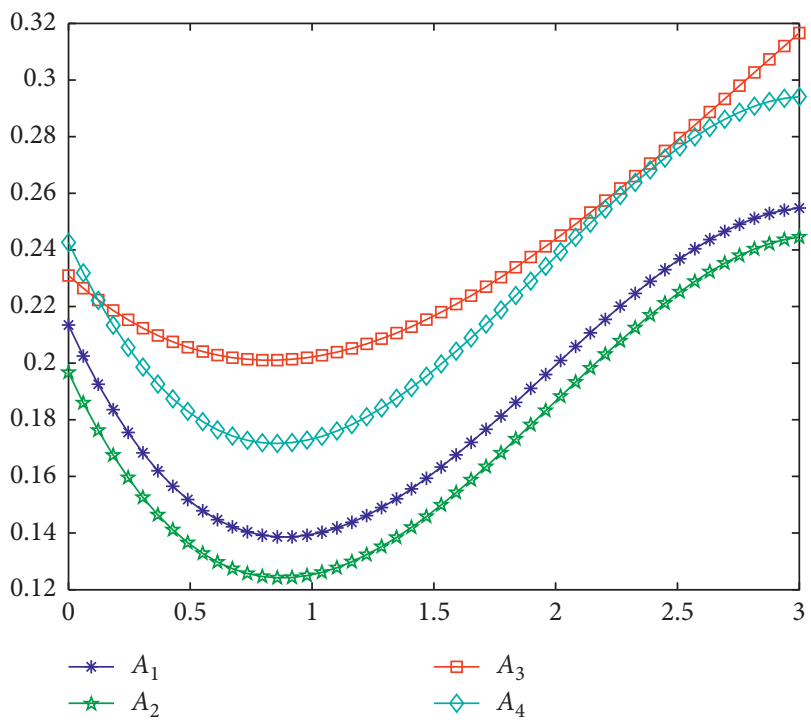

FIgURE 5: Variation of SC $\left(\mathscr{F}_{r}\right)$ based on the A-HF2TLWBM operator $(p=1, q \in(0,3))$.

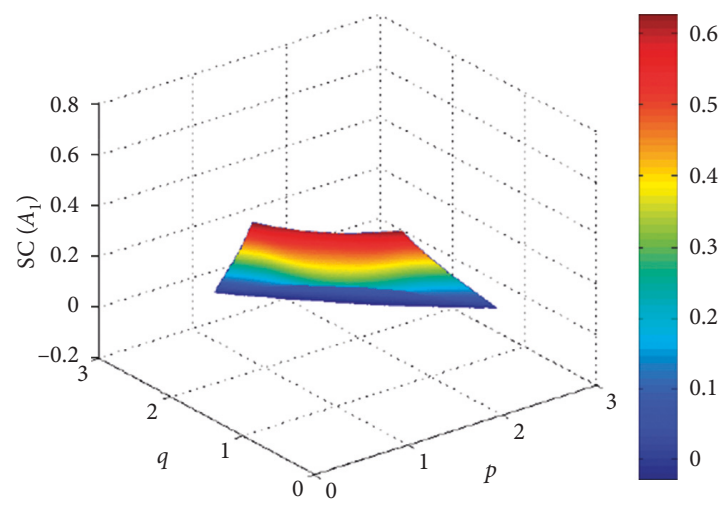

Figure 6: SC $\left(\mathscr{F}_{1}\right)$ of $A_{1}$ based on the A-HF2TLWGBM operator.

A-HF2TLWBM operator with the smaller parameter values $p$ and $q$, and an optimistic decision-maker can use the AHF2TLWGBM operator to select the larger parameter 


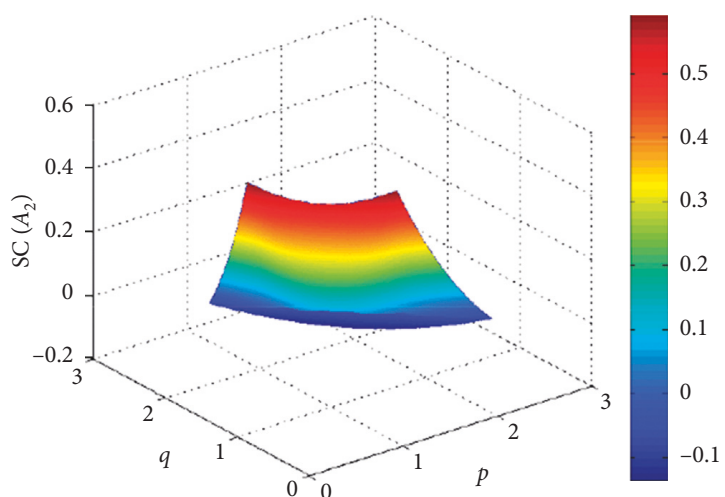

Figure 7: SC $\left(\mathscr{F}_{2}\right)$ of $A_{2}$ based on the A-HF2TLWGBM operator.

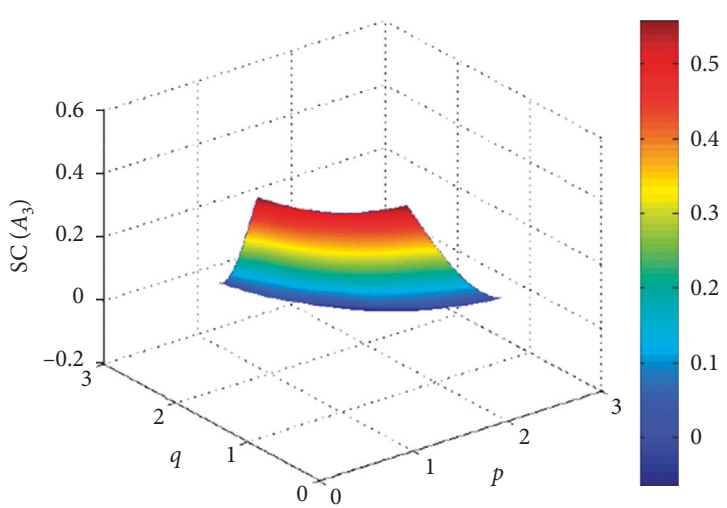

Figure 8: SC $\left(\mathscr{F}_{3}\right)$ of $A_{3}$ based on the A-HF2TLWGBM operator.

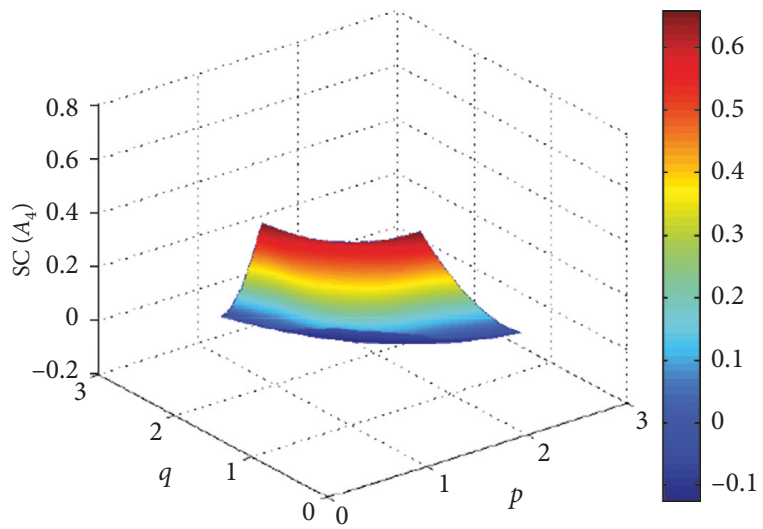

Figure 9: SC $\left(\mathscr{F}_{4}\right)$ of $A_{4}$ based on the A-HF2TLWGBM operator.

values. Neutral decision making results are obtained using the arithmetic averages of the score values $\mathrm{SC}\left(\mathscr{F}_{r}\right)$ as shown in Figures 11-14.

6.4. Comparison Analysis with the Existing Methods. To illustrate the practicality and advantages of the proposed method, we compare the proposed MCDM approach with the existing methods $[12,13,25,26]$.

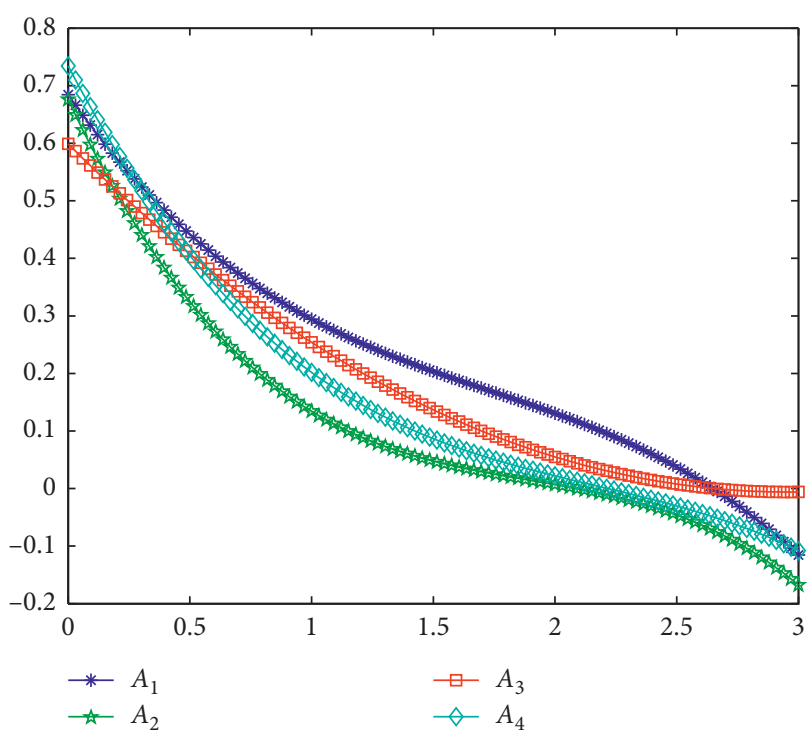

Figure 10: Variation of SC $\left(\mathscr{F}_{r}\right)$ based on the A-HF2TLWGBM operator $(p=1, q \in(0,3))$.

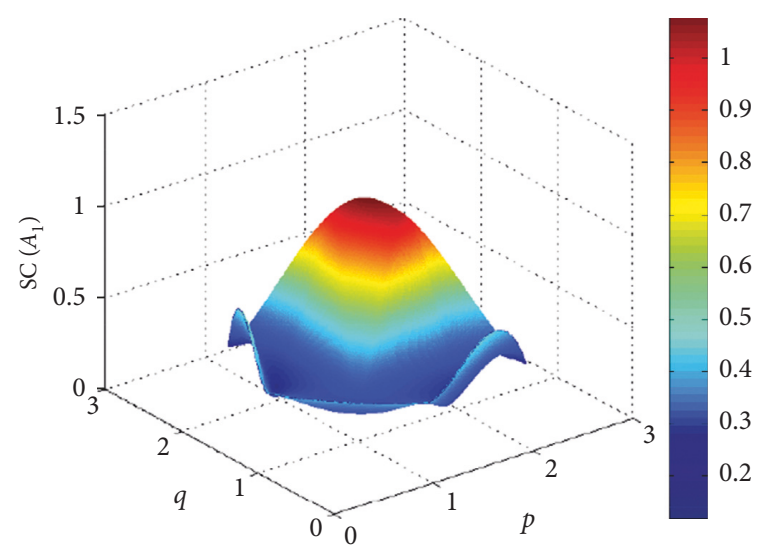

FIgURE 11: SC $\left(\mathscr{F}_{1}\right)$ of $A_{1}$ based on the A-HF2TLWBM operator and the A-HF2TLWGBM operator.

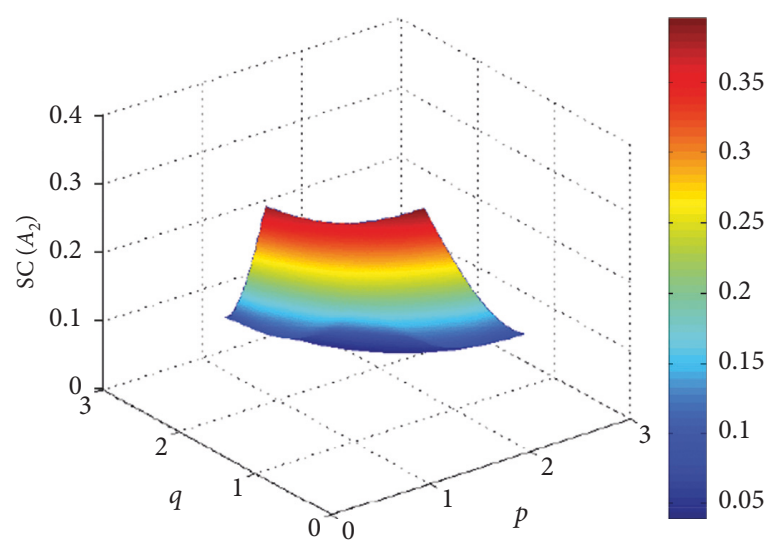

FIgURE 12: SC $\left(\mathscr{F}_{2}\right)$ of $A_{2}$ based on the A-HF2TLWBM operator and the A-HF2TLWGBM operator. 


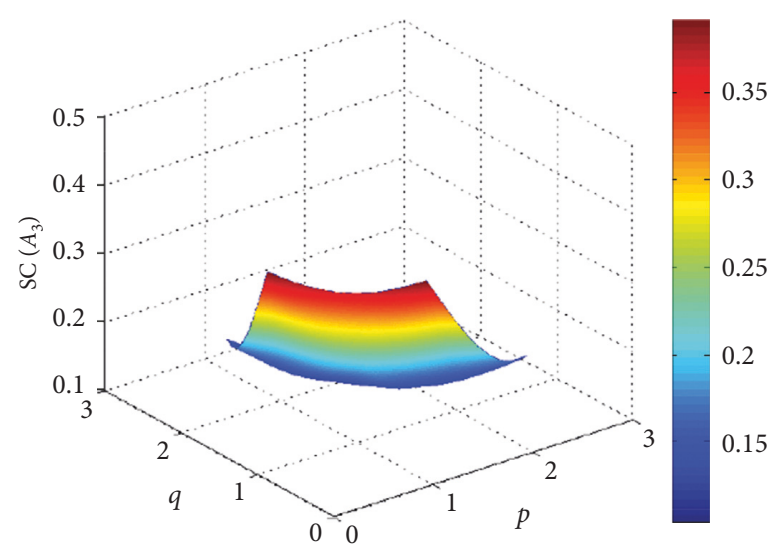

Figure 13: SC $\left(\mathscr{F}_{3}\right)$ of $A_{3}$ based on the A-HF2TLWBM operator and the A-HF2TLWGBM operator.

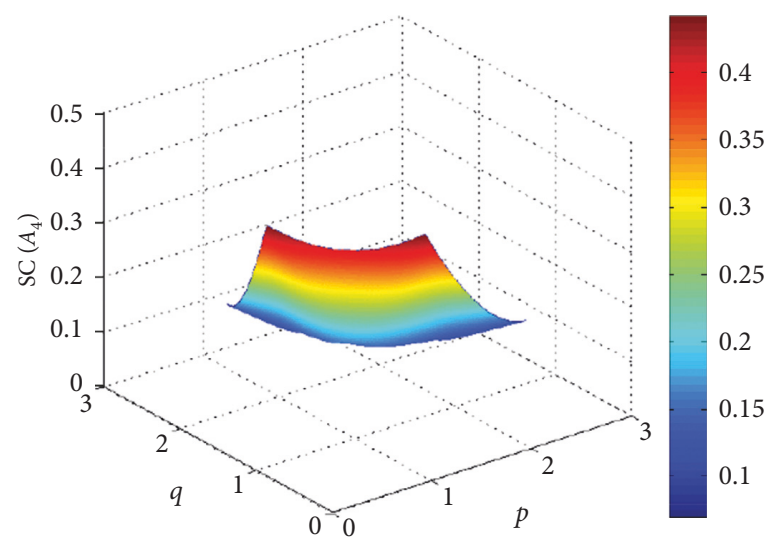

Figure 14: SC $\left(\mathscr{F}_{4}\right)$ of $A_{4}$ based on the A-HF2TLWBM operator and the A-HF2TLWGBM operator.

6.4.1. Comparison Analysis of the MCDM Method with DHFS Information. For comparing the proposed MCDM approach with the existing method [26], we use the same practical MCDM problem decision background as shown in Table 2 and the same criteria weight vector $(0.2,0.1,0.3,0.4)$. We apply the DHFWGBM operator and DHFCGBM operator conceived in [26] to cope with Table 2 without the linguistic terms. The corresponding dual hesitant fuzzy matrix is shown in Table 3. The obtained ranking result of alternatives is $A_{4}>A_{2}>A_{3}>A_{1}$ when $m_{1}=m_{2}=1$. We also identify the optimal alternative(s) by applying the approach designed in Section 5, where the A-HF2TLWGBM operator with $p=q=1, \zeta(c)=-\log c$, and $\chi(c)=-\log (1-c)$ is employed to fuse all preference values. The ranking order of alternatives is also $A_{4}>A_{2}>A_{1}>A_{3}$. The differences of two ranking results arise from the difference of the interrelationship of the input variables. Through comparison with the existing method [26], we observe that when $\zeta(c)=$ $-\log c$ and $\chi(c)=-\log (1-c)$, the DHFWGBM operator is the general form of A-HF2TLWGBM operator. The ranking result by using the DHFWGBM operator [26] is the same as our ranking result. The established methods are two parameterized families of the aggregation operators of HF2TLSs, which cover a wide range of particular forms that exhibit a more flexible way of adapting the specific needs of problem solving.
6.4.2. Comparison Analysis with the Existing Methods. In the literature, there are some concepts and aggregation operators involving Fermatean fuzzy information. Senapti and Yager $[12,13]$ proposed an approach for dealing with the MCDM problem with Fermatean fuzzy information by establishing some aggregation operators. Liu et al. [25] presented the FFLWA operator, the FFLWG operator, and the Fermatean fuzzy linguistic distance measures to address MCDM problems with FFLTSs. Jamil and Rashid [26] developed the dual hesitant fuzzy geometric Bonferroni mean method for handling the MCDM problems with DHFSs. Although these concepts and methods are able to improve the ability of representing and processing complex data from different perspectives, they fail to deal with HF2TLTSs information systems. Compared with the method in [26], the pair of membership degree $\mu(z)$ and nonmembership degree $\nu(z)$ are involved in two methods; however, they perform difference roles. We observe that the method in [26] adopts DHFSs, and the preference information is characterized by the pair of membership degree $\mu(z)$ and nonmembership degree $\nu(z)$ with $\mu(z)+\nu(z) \leq 1$. It can only solve the MCDM problem described by using $\mu(z)$ and $\nu(z)$. However, our new concept of HF2TLSs by combining the FFS and 2-tuple linguistic terms, in which $\mu(z)$ and degree $\nu(z)$ are with a more loose constraint $0 \leq\left(\mu_{F}(z)\right)^{3}+\left(\nu_{F}(z)\right)^{3} \leq 1$, is 


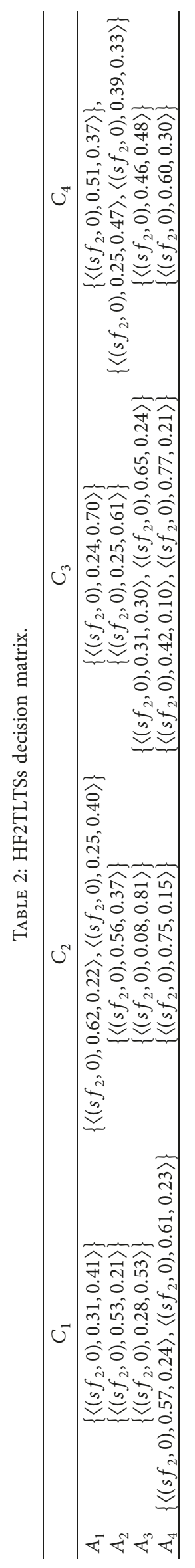


Table 3: Dual hesitant fuzzy matrix.

\begin{tabular}{ccccc}
\hline & $C_{1}$ & $C_{2}$ & $C_{3}$ & $C_{4}$ \\
\hline$A_{1}$ & $(\{0.31\},\{0.41\})$ & $(\{0.62,0.25\},\{0.22,0.40\})$ & $(\{0.24\},\{0.70\})$ & $(\{0.51\},\{0.37\})$ \\
$A_{2}$ & $(\{0.53\},\{0.21\})$ & $(\{0.56\},\{0.37\})$ & $(\{0.25\},\{0.61\})$ & $(\{0.25,0.39\},\{0.47,0.33\})$ \\
$A_{3}$ & $(\{0.28\},\{0.53\})$ & $(\{0.08\},\{0.81\})$ & $(\{0.31,0.65\},\{0.30,0.24\})$ & $(\{0.46\},\{0.48\})$ \\
$A_{4}$ & $(\{0.57,0.61\},\{0.24,0.23\})$ & $(\{0.75\},\{0.15\})$ & $(\{0.42,0.77\},\{0.10,0.21\})$ & $(\{0.60\},\{0.30\})$ \\
\hline
\end{tabular}

employed to represent the uncertainty of the linguistic preference information. HF2TLSs can better align with human decision making presentation and characterize more complex uncertainty information.

\section{Conclusions}

To further characterize and handle decision-makers' hesitancy and uncertainty in some complex MCDM problems, this study introduces a new class of uncertain linguistic variables called hesitant Fermatean 2-tuple linguistic sets (HF2TLSs). We extend the ATS and BM operators to the hesitant Fermatean 2-tuple linguistic environment and further proffer some special operational laws for HF2TLSs, by which the A-HF2TLWBM operator and the AHF2TLWGBM operator are developed. These operators offer an efficient tool in practical applications by capturing the interrelationship of the input variables and avoiding information loss. In addition, we further prove their idempotency, boundedness, and monotonicity. We also discuss some special cases and compare our approach to the existing methods. An example demonstrates the method's practicality and effectiveness with a detailed discussion under different influences of the parameters $p$ and $q$. In future research, we will focus on solving multicriteria group decision making problems under the background of HF2TLSs.

\section{Data Availability}

The experts' preferences data used to support the findings of this study are included within the article.

\section{Conflicts of Interest}

The authors declare that they have no conflicts of interest.

\section{Acknowledgments}

This work was supported by the Natural Science Foundation of China (Grant nos. 61803065 and 61773352) and the Fundamental Research Funds for the Central Universities (Grant nos. 3132019175 and 3132019323).

\section{References}

[1] J. Ye, "Some weighted aggregation operators of trapezoidal neutrosophic numbers and their multiple attribute decision making method," Neutrosophic Sets and Systems, vol. 28, no. 2, pp. 387-402, 2015.

[2] K. Alhazaymeh, M. Gulistan, M. Khan, and S. Kadry, "Neutrosophic cubic einstein hybrid geometric aggregation operators with application in prioritization using multiple attribute decision-making method," Mathematics, vol. 7, no. 4, p. 346, 2019.

[3] C. Fan, J. Ye, S. Feng, E. Fan, and K. Hu, "Multi-criteria decision-making method using Heronian mean operators under a Bipolar neutrosophic environment," Mathematics, vol. 7, no. 1, p. 97, 2019.

[4] Y. X. Ma, J. Q. Wang, J. Wang, and X. H. Wu, “An interval neutrosophic linguistic multi-criteria group decision-making method and its application in selecting medical treatment options," Neural Computing \& Applications, vol. 28, no. 9, pp. 2745-2765, 2016.

[5] L. A. Zadeh, "The concept of a linguistic variable and its application to approximate reasoning-III," Information Sciences, vol. 9, no. 1, pp. 43-80, 1975.

[6] L. A. Zadeh, "Fuzzy sets," Information \& Control, vol. 8, no. 3, pp. 338-353, 1965.

[7] K. T. Atanassov, "Intuitionistic fuzzy sets," Fuzzy Sets Systems, vol. 20, no. 1, pp. 87-96, 1986.

[8] R. R. Yager, "Pythagorean membership grades in multicriteria decision making," IEEE Transactions on Fuzzy Systems, vol. 22, no. 4, pp. 958-965, 2014.

[9] X. Zhang and Z. Xu, "Extension of TOPSIS to multiple criteria decision making with Pythagorean fuzzy sets," International Journal of Intelligent Systems, vol. 29, no. 12, pp. 1061-1078, 2014.

[10] S. Z. Zeng, Z. M. Mu, and T. Balezentis, “A novel aggregation method for Pythagorean fuzzy multiple attribute group decision making," Journal of Intelligent \& Fuzzy Systems, vol. 33, no. 3, pp. 573-585, 2018.

[11] R. M. Rodriguez, L. Martinez, and F. Herrera, "Hesitant fuzzy linguistic term sets for decision making," IEEE Transactions on Fuzzy Systems, vol. 20, no. 1, pp. 109-119, 2012.

[12] T. Senapati and R. R. Yager, "Fermatean fuzzy sets," Journal of Ambient Intelligence and Humanized Computing, vol. 85, pp. 1-12, 2019.

[13] T. Senapati and R. R. Yager, "Fermatean fuzzy weighted averaging/geometric operators and its application in multicriteria decision-making methods," Engineering Applications of Artificial Intelligence, vol. 85, pp. 112-121, 2019.

[14] L. Martínez, R. M. Rodriguez, and F. Herrera, "Linguistic approaches based on the 2-tuple fuzzy linguistic representation model," The 2-tuple Linguistic Model, vol. 20, no. 1, pp. 43-50, 2015.

[15] D. K. Joshi, I. Beg, and S. Kumar, "Hesitant probabilistic fuzzy linguistic sets with applications in multi-criteria group decision making problems," Mathematics, vol. 6, no. 4, p. 47, 2018.

[16] F. Herrera and L. Martínez, "A 2-tuple fuzzy linguistic representation model for computing with words," IEEE Transactions on Fuzzy Systems, vol. 8, no. 6, pp. 746-752, 1999.

[17] F. Herrera and L. Martínez, "A model based on linguistic 2tuples for dealing with multigranular hierarchical linguistic contexts in multi-expert decision-making," IEEE Transactions on Systems Man Cybernetics-Systems, vol. 31, no. 2, pp. 227234, 2000. 
[18] W.-P. Wang, "Evaluating new product development performance by fuzzy linguistic computing," Expert Systems with Applications, vol. 36, no. 6, pp. 9759-9766, 2009.

[19] W. Zhang, Y. Xu, and H. Wang, "A consensus reaching model for 2-tuple linguistic multiple attribute group decision making with incomplete weight information," International Journal of Systems Science, vol. 47, no. 2, pp. 389-405, 2016.

[20] V. Torra, "Hesitant fuzzy sets," International Journal of Systems Science, vol. 25, pp. 529-539, 2010.

[21] W. Zhou, Z. Xu, and M. Chen, "Preference relations based on hesitant-intuitionistic fuzzy information and their application in group decision making," Computers \& Industrial Engineering, vol. 87, pp. 163-175, 2015.

[22] T. Rashid, S. Faizi, Z. S. Xu, and S. Zafar, "Electre-based outranking method for multi-criteria decision making using hesitant intuitionistic fuzzy linguistic term sets," Notes on Instuitionistic Fuzzy Sets, vol. 20, no. 12, pp. 1-15, 2017.

[23] L. Zhang, F. Meng, and B. Meng, "A new procedure to intuitionistic uncertain linguistic group decision making," Informatica, vol. 29, no. 2, pp. 371-397, 2018.

[24] G. Wei, M. Lu, and F. E. Alsaadib, "Pythagorean 2-tuple linguistic aggregation operators in multiple attribute decision making," Journal of Intelligent \& Fuzzy Systems, vol. 2, pp. 1-13, 2017.

[25] D. Liu, Y. Liu, and X. Chen, "Fermatean fuzzy linguistic set and its application in multicriteria decision making," International Journal of Intelligent Systems, vol. 34, no. 5, pp. 878-894, 2019.

[26] R. N. Jamil and T. Rashid, "Application of dual hesitant fuzzy geometric Bonferroni mean operators in deciding an energy policy for the society," Mathematical Problems in Engineering, vol. 2018, 14 pages, 2018.

[27] X. Liu, Z. Tao, H. Chen, and L. Zhou, "A new interval-valued 2-tuple linguistic Bonferroni mean operator and its application to multiattribute group decision making," International Journal of Fuzzy Systems, vol. 19, no. 1, pp. 86-108, 2017.

[28] P. Liu and W. Liu, "Multiple-attribute group decision-making based on power Bonferroni operators of linguistic $q$-rung orthopair fuzzy numbers," International Journal of Intelligent Systems, vol. 34, no. 4, pp. 652-689, 2019.

[29] R. R. Yager, “On generalized Bonferroni mean operators for multi-criteria aggregation," International Journal of Approximate Reasoning, vol. 50, no. 5, pp. 1279-1286, 2009.

[30] G. Beliakov, S. James, J. Mordelová, T. Rückschlossová, and R. R. Yager, "Generalized Bonferroni mean operators in multi-criteria aggregation," Fuzzy Sets and Systems, vol. 161, no. 17 , pp. 2227-2242, 2010.

[31] M. Xia, Z. Xu, and B. Zhu, "Geometric Bonferroni means with their application in multi-criteria decision making," Knowledge-Based Systems, vol. 40, no. 1, pp. 88-100, 2013.

[32] M. Xia, Z. Xu, and B. Zhu, "Generalized intuitionistic fuzzy Bonferroni means," International Journal of Intelligent Systems, vol. 27, no. 1, pp. 23-47, 2011.

[33] B. Zhu, Z. S. Xu, and M. M. Xia, "Hesitant fuzzy geometric Bonferroni means," Information Sciences, vol. 205, no. 1, pp. 72-85, 2010.

[34] R. T. Zhang, J. Wang, X. M. Zhu, M. M. Xia, and M. Yu, "Some generalized pythagorean fuzzy bonferroni mean aggregation operators with their application to multiattribute group decision-making," Complexity, vol. 2017, Article ID 5937376, 16 pages, 2017.
[35] G. J. Klir and B. Yuan, Yuan, Fuzzy sets and fuzzy logic: Theory andapplications, vol. 1, Prentice-all, Upper Saddle River, NJ, USA, 1995.

[36] H. T. Nguyen and E. A. Walker, "A first course in fuzzy logic," Mathematical Gazette, vol. 81, p. 491, 2005.

[37] P. Liu, "The aggregation operators based on archimedean $t$ conorm and $t$-norm for single-valued neutrosophic numbers and their application to decision making," International Journal of Fuzzy Systems, vol. 18, no. 5, pp. 849-863, 2016.

[38] J. Lan, Q. Sun, Q. Chen, and Z. Wang, "Group decision making based on induced uncertain linguistic OWA operators," Decision Support Systems, vol. 55, no. 1, pp. 296-303, 2013.

[39] M. Xia, Z. Xu, and B. Zhu, "Some issues on intuitionistic fuzzy aggregation operators based on Archimedean $t$-conorm and $t$ norm," Knowledge-Based Systems, vol. 31, pp. 78-88, 2012.

[40] Z. Tao, H. Chen, L. Zhou, and J. Liu, "On new operational laws of 2-tuple linguistic information using Archimedean $t$-norm and s-norm," Knowledge-Based Systems, vol. 66, pp. 156-165, 2014. 


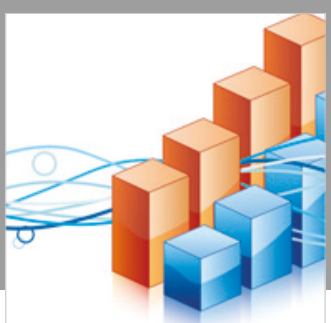

Advances in

Operations Research

\section{-n-m}
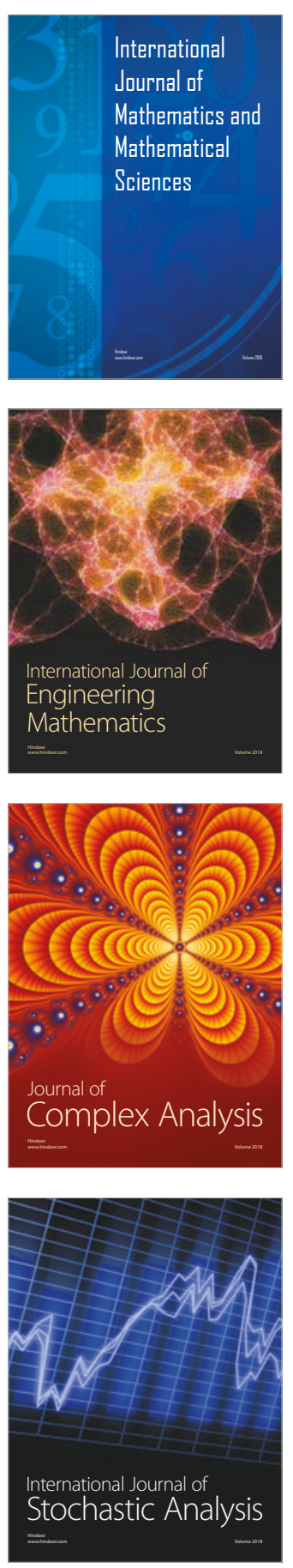
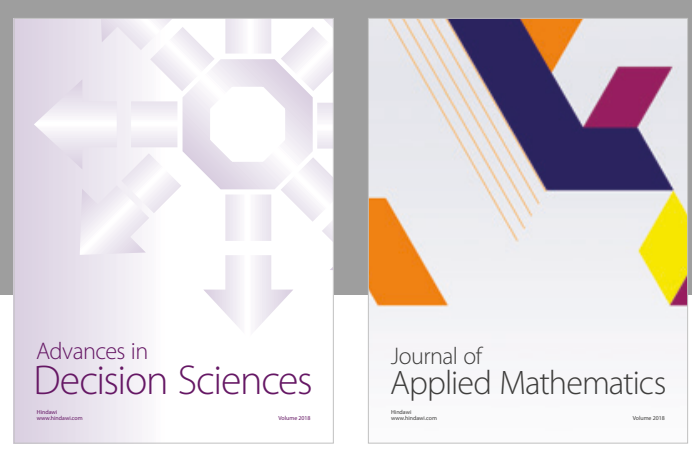

Journal of

Applied Mathematics
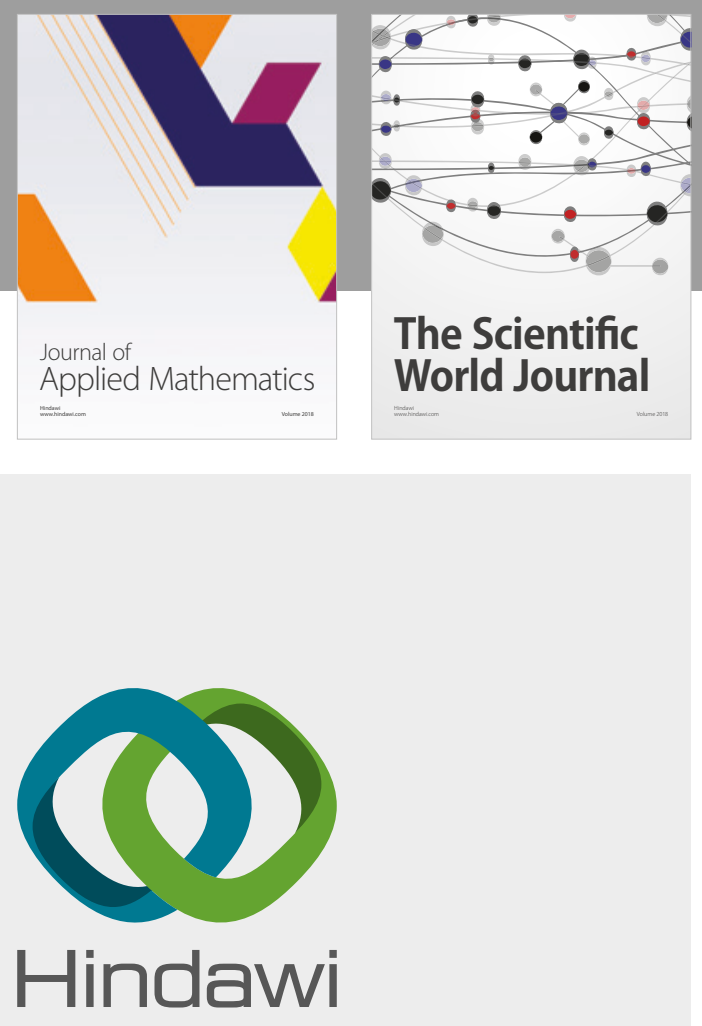

Submit your manuscripts at

www.hindawi.com

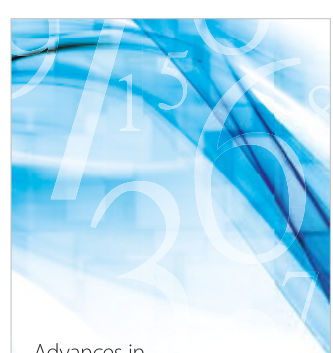

Advances in
Numerical Analysis
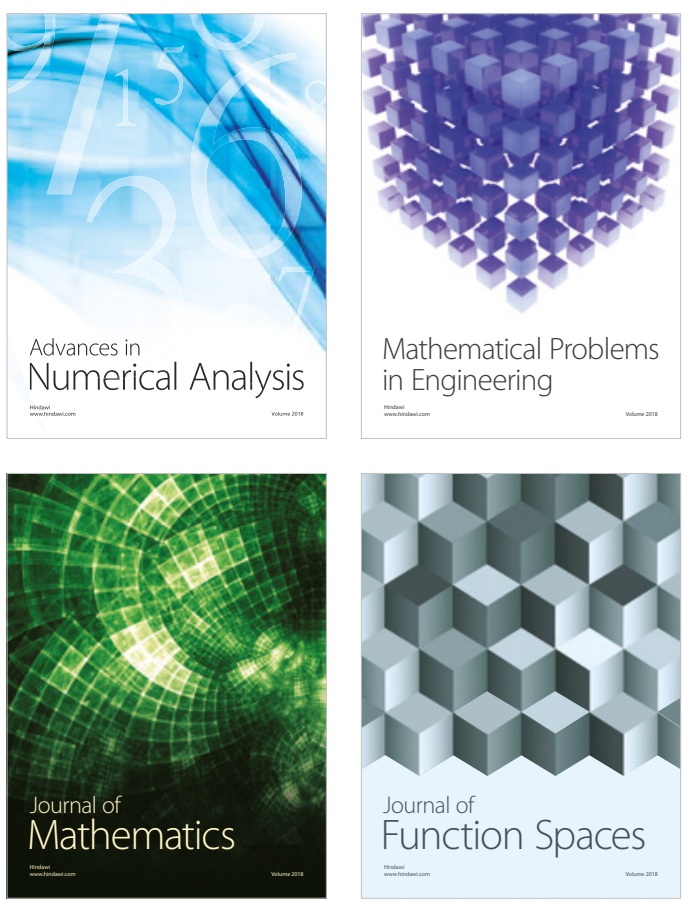

Mathematical Problems in Engineering

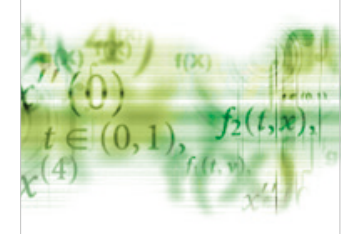

International Journal of

Differential Equations

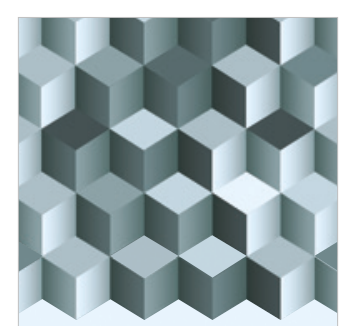

Journal of

Function Spaces
The Scientific

World Journal

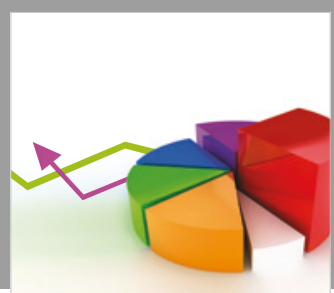

Journal of

Probability and Statistics
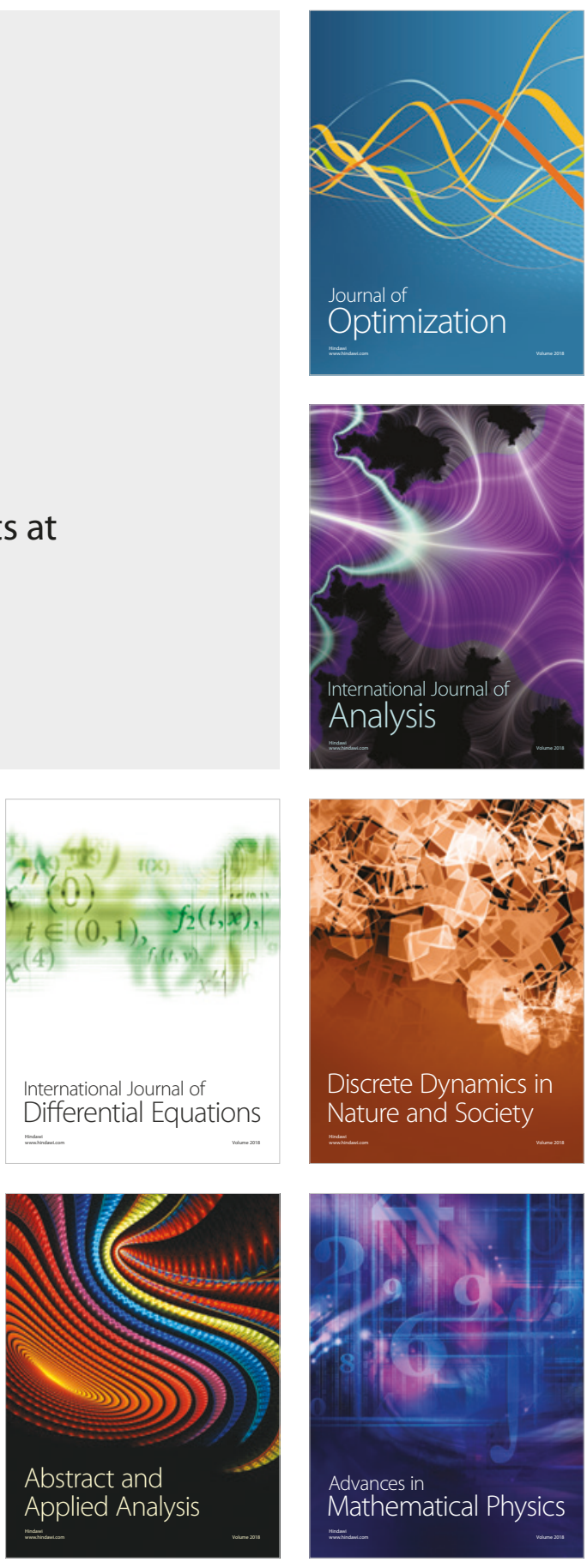